\title{
Il terremoto della Valnerina del 19 Settembre 1979 Indagine Macrosismica
}

(The Valnerina earthquake of September 19th, 1979).

Macroseismic investigation

\author{
P. Favali - L. Giovani - M.C. SPadea - M. Vecchi
}

Ricevuto il 13 giugno 1980

\begin{abstract}
RIASSUNTO
Nel presente lavoro si espongono i risultati dell'indagine macrosismica condotta in occasione del terremoto della Valnerina del 19 Settembre 1979. E' stato possibile determinare l'andamento del campo macrosismico tino all'isosista di $\mathrm{V}$ grado, nonché i relativi parametri ipocentrali. Dal loro esame si evidenziano le caratteristiche superficiali dello evento. Infine è stata studiata l'attenuazione calcolando i coefficienti sia nella direzione di massimo (N-S) che di minimo (E-W) allungamento.
\end{abstract}

\section{ABSTRact}

In this paper the results of macroseismic investigation concerning the Valnerina carthquake (september 19, 1979) have been carried out. It 
was pcssible to define the trend of macroseismic field until the isoseismal cf $\mathrm{V}$ degree (MCS), as well as the relative hypocentral parameters, and to point out the superficial characteristics of the event. Finally the attenuation has been studied computing the coefficients, both of the elongation in maximum direction (N-S) and in minimum (E-W) one.

\section{1 - Premessa}

La sera del 19 Settembre alle ore $23^{\mathrm{h}} 35^{\mathrm{m}}$, ora legale italiana, gli strumenti sismici italiani e non, registrarono una scossa di terremoto che fin dalle prime determinazioni dell'epicentro e della Magnitudo dimostrava aver colpito un'area molto prossima a Norcia con carattere "disastroso » ed una intensità epicentrale $\left(I_{\mathrm{v}}\right)$ valutabile intorno all'VIII grado della scala Mercalli-CancaniSieberg.

Le prime notizie giunte dalla zona interessata, sia pur frammentarie, confermavano l'entità del fenomeno in tutta la sua gravità. In seguito a ciò, fin dalla mattina del 20 Settembre, gli AA. decidevano di intervenire per effettuare un rilevamento macrosismico nell'area colpita. Questo nel tentativo di non perdere gli effetti della scossa principale, ed allo scopo di superare le ambiguità di valutazione riscontrabili nello studio di eventi passati, basato esclusivamente su fonti di carattere storico.

\section{2 - DATI}

Il materiale utilizzato proviene da fonti diverse. Sono stati visitati 201 paesi delle province di Rieti, Terni, Perugia, Ascoli Piceno e L'Aquila al fine di delimitare l'area di massimo scuotimento. I comuni e le frazioni compresi nell'area megasismica sono stati oggetto di una approfondita indagine. Inoltre, affinché la classificazione dei danni alle strutture risultasse la più oggettiva possibile, sono stati considerati i dati che il Centro di coordinamento di Norcia aveva raccolto nell'area dei comuni della Valnerina fin dal 28 settembre. Il metodo adottato per la definizione dei danni ne permette una valutazione secondo 4 tipi: leggere lesioni, danni medi, gravi e demolizioni (crolli parziali 
e/o totali). Infine sono stati elaborati oltre 500 questionari macrosismici pervenuti all'I.N.G. dalle Regioni: Umbria, Marche, Lazio, Abruzzo, Molise, Toscana, tramite i punti stazione dei Carabinieri (All. 1). Ciò è stato possibile a seguito di un accordo che l'Istituto Nazionale di Geofisica, fin dal 1978, ha stipulato con il Comando Generale dell'Arma dei CC.

Questo si è reso necessario al fine di istituire un nuovo servizio continuativo di raccolta dati macrosismici in sostituzione del precedente, curato dall'Ufficio Centrale di Meteorologia e Geodinamica fino al 1975. Nell'Allegato 2 diamo un esempio del tipo di cartoline che venivano usate.

\section{3 - Metonologia}

Il criterio seguito durante il rilevamento macrosismico è stato quello di avvicinarsi progressivamente e lungo raggi diversi dalla "periferia " alla ipotizzata zona epicentrale. I motivi che hanno portato a questa decisione sono molteplici. Innanzi tutto ciò è necessario per riuscire a delimitare nel più breve tempo possibile l'area epicentrale, non avendo sempre l'immediata conoscenza della reale situazione, come del resto è avvenuto nel caso in questione. In secondo luogo, trovandosi di fronte ad un periodo sismico di grande portata, si rende necessario acquisire prima quei dati che riguardano zone limitrofe di intensità intermedia, che possono lasciare dubbi maggiori se raccolti in ritardo, e infine i dati provenienti dall'area epicentrale. Infatti, limitatamente a fenomeni di questo tipo, gli effetti delle repliche difficilmente tendono a sommarsi a quelli della scossa principale o comunque non sono tali da modificarne sostanzialmente la valutazione all'epicentro. Per l'analisi degli effetti provocati dal terremoto nell'area megasismica, ci siamo avvalsi delle mappe catastali in scala 1:2000 dei comuni interessati in modo d'avere la planimetria degli abitati. Queste mappe hanno permesso di avere una percezione visiva della distribuzione areale del danno che, associata alla tipologia delle singole costruzioni ( $v$. tabella 1), consente una dettagliata determinazione delle percentuali di danno. Nel corso della campagna gli AA. hanno constatato la necessità e l'utilità di avvalersi 
di un questionario sintetico (vedi allegato 3) per mezzo del quale si potevano acquisire tutte le informazioni relative agli effetti per ciascuna località. L'informazione raccolta direttamente permette un'analisi geologica e strutturistica più dettagliata che non attraverso dati indiretti. Anzi dà anche la possibilità di valutare appieno il peso di questi ultimi.

\section{TABELLA 1}

Classificazione dei tipi di costruzione $\left({ }^{*}\right)$

A) Costruzioni in pietra grezza, mattoni cotti legati con malte povere, vecchie mal ristrutturate con soffitti camorcanne o a travature in legno (scadenti).

B) Costruzioni in pietra squadrata, mattoni comuni, tufo, legati con buone malte, con soffitti a travatura in ferro o in legno (mediocri).

C) Costruzioni in muratura cordolate, ben ristrutturate (quasi buone).

D) Case in legno ben progettate, in resine, prefabbricati, costruzioni in cemento armato, strutture antisismiche (buone, ottime).

\section{4 - Quadro GeOlogico}

L'area megasismica fa parte, dal punto di vista geologico, delle strutture plicative della facies Umbro-Marchigiana di cui ne costituisce la parte meridionale. In questa zona si trovano $\mathrm{i}$ grandi fronti di sovra-scorrimento vergenti verso l'Adriatico (Monti Sibillini) che sono in contatto di tipo tettonico con facies di transizione alla Laziale-Abruzzese. La struttura a pieghe

(*) Per necessità, data la diversità dei tipi di strutture, gli AA. hanno ritenuto utile suddividere le costruyioni in $4 \mathrm{tipi}$, anzichè in 3 come fatto nella scala MCS ed MSK (vedi All. 3). 
nella zona nursina ha subito degli sbloccamenti associati ad una serie di faglie inverse e a fronti di sovrascorrimento che hanno determinato una serie di altopiani (Piana di S. Scolastica, Piano di Castelluccio).

Dal punto di vista stratigrafico si ritrovano intercalazioni di calcari, marne anche selcifere ed argille, caratterizzanti gli uni ambiente marino di piattaforma, e le altre formazioni più pelagiche.

\section{5 - Campo Macrosismico}

Dall'elaborazione di tutti i dati disponibili, abbiamo potuto definire l'andamento del campo macrosismico dall'area megasismica a quella di $\mathrm{V}$ grado, rappresentato in fig. 1 nella scala 1:500000. Esaminando il campo possiamo notare una prevalente propagazione in direzione N-S; mentre la massima attenuazione è riscontrabile lungo la E-W relativamente alle maggiori intensità fino al VI grado. Nella fig. 1 non abbiamo potuto materialmente riportare tutte le località di cui avevamo informazioni, comunque l'elenco completo compare in tabella 2. L'area di massimo scuotimento è stata oggetto di un'indagine approfondita, essendo stato condotto il rilevamento in maniera capillare su tutto il territorio dei comuni interessati, comprese le frazioni. I risultati sono riportati nella fig. 2 in scala 1:100.000. I dati da noi raccolti sono stati integrati dai rilievi effettuati dal Centro di Coordinamento di Norcia. Questo ha permesso di determinare per ogni comune e frazione sia la tipologia delle costruzioni che le percentuali di danno riscontrate (vedi tab. 3 ).

Il comune di Norcia è stato ulteriormente diviso in 6 zone: (1, centro storico; 2, zona sopra l'Hotel Europa; 3, da metà Corso a fuori porta; 4, zona Hotel Europa; 5, parte strada statale da Roma; 6, zona FIAT).

Fin dai primi rilievi è stato possibile constatare che all'interno dell'isosisma di VIII grado esisteva un'area in cui gli effetti macrosismici avevano avuto un'ulteriore esaltazione. In seguito a ciò, è stata tracciata una isosista di VIII-IX, al solo scopo di evidenziare questo fenomeno (fig. 2). 


\section{6 - DeFinizione dei PARAMETRI}

Sono stati calcolati per ogni isosisma l'area ed il raggio del cerchio equivalente, nonché l'epicentro e la profondità ipocentrale macrosismici; i risultati sono riportati in Tab. 4.

\section{Area di massimo scuotimento}
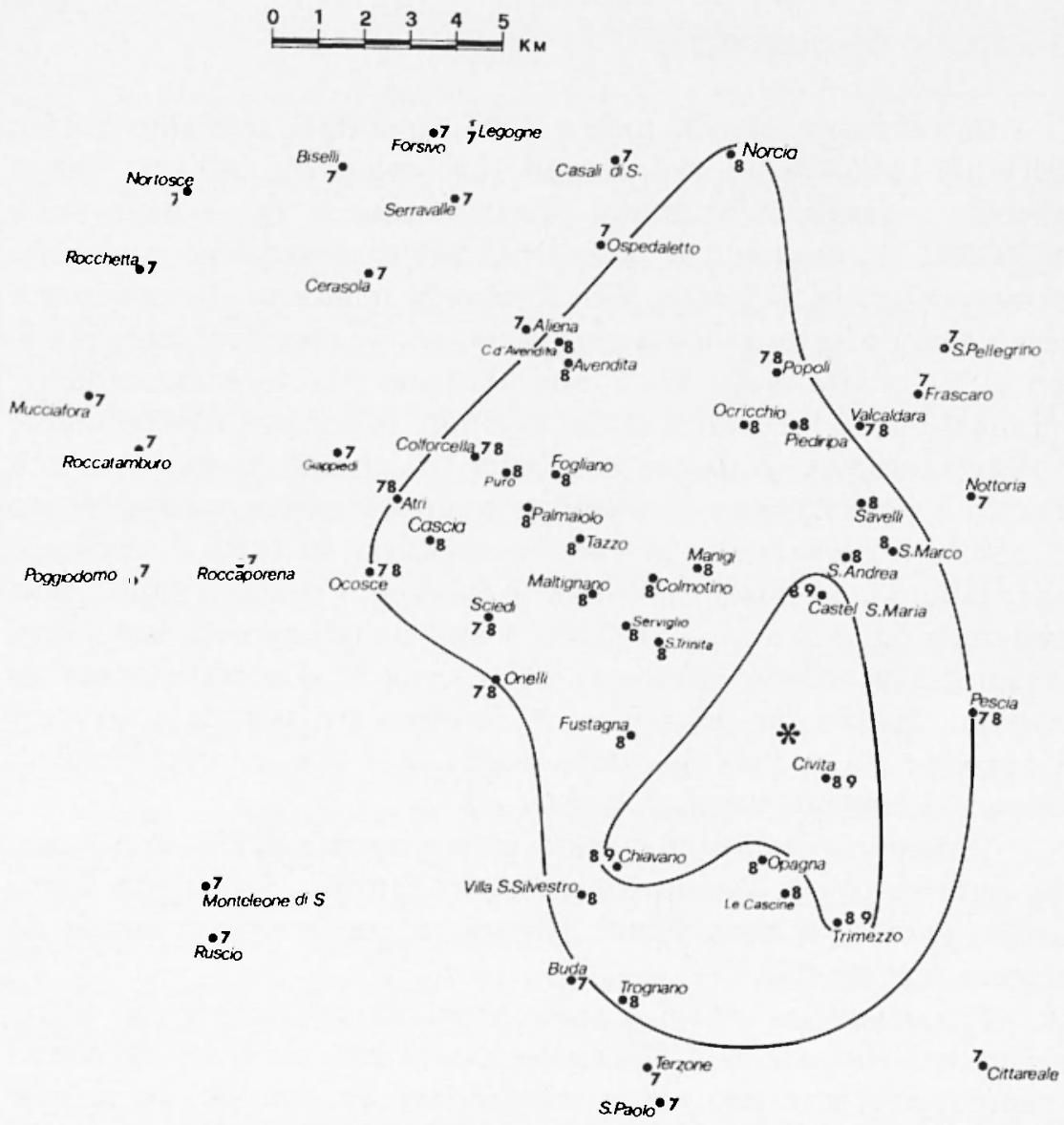

Fig. 2 - Mappa dell'isosista $I_{n}=$ VIII-IX, I = VIII (scala $1: 100000$ ). 


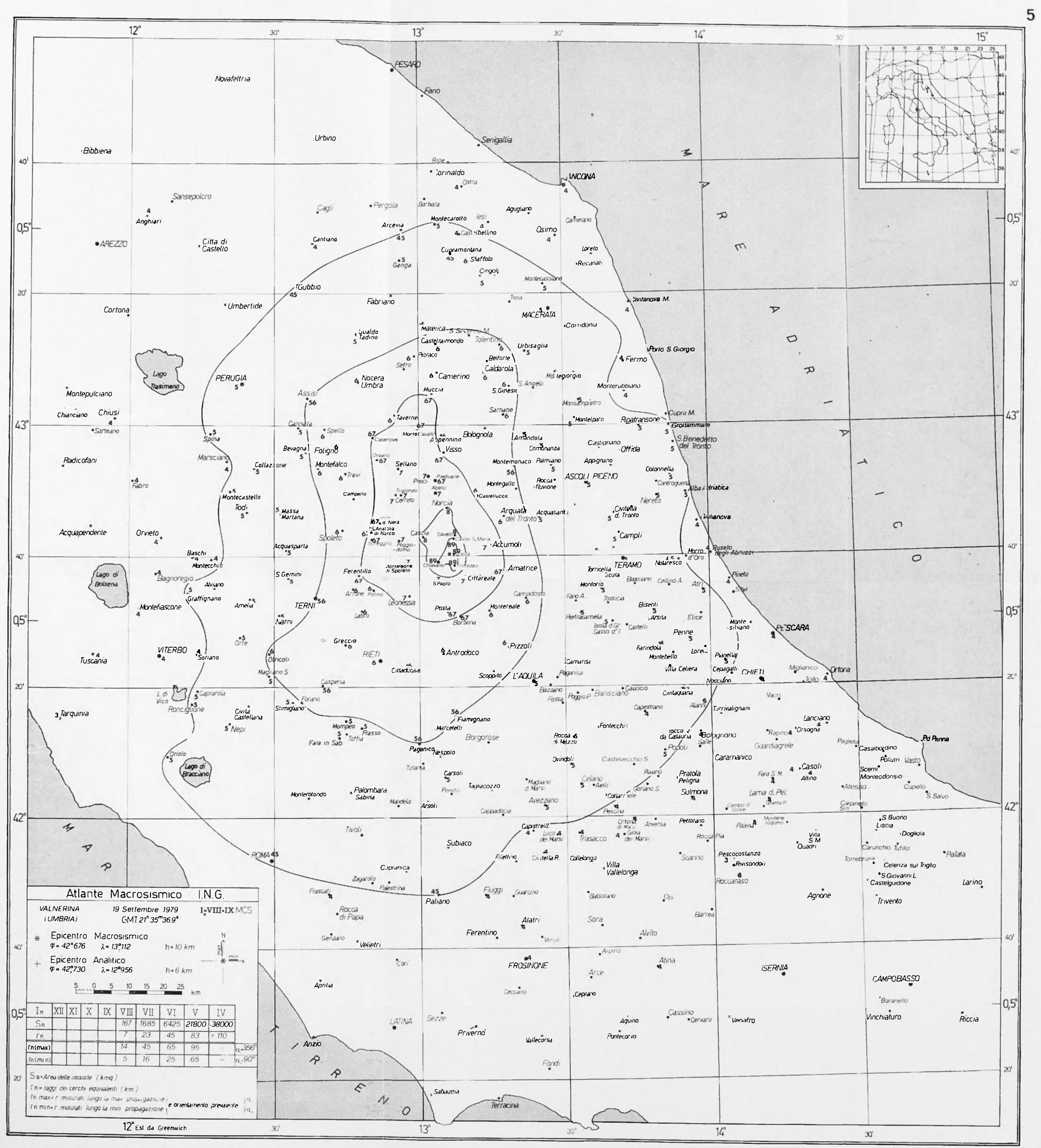

Fig. 1 - Mappacompleta delle isosiste (scala 1:500000) 
TABELLA 4

Parametri macrosismici

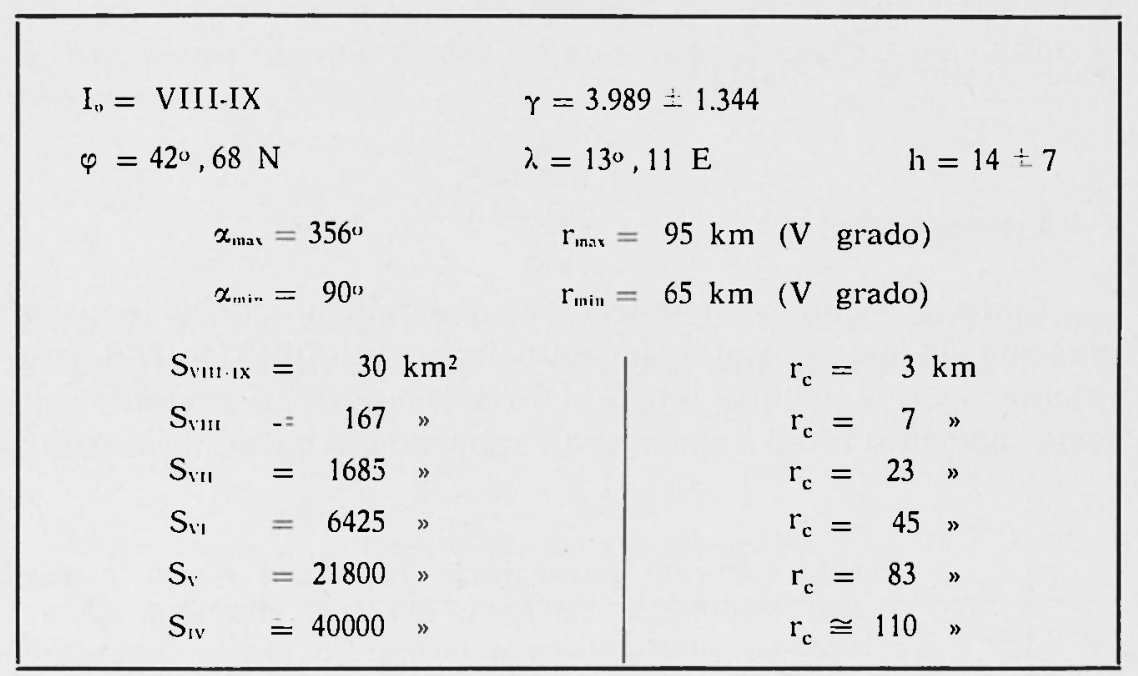

L'epicentro macrosismico è rappresentato dal baricentro dell'area megasismica, le cui coordinate sono state ricavate dalla carta geografica della zona in scala 1: 100.000 .

La profondità ipocentrale, mediata tra più valori, è stata stimata mediante la formula:

$$
h=\frac{\left(r_{c}\right)_{n}}{\left[10 \frac{2\left(I_{0}-I_{n}\right)}{\gamma}-1\right]^{1 / 2}}
$$

dove $r_{c}$ rappresenta il raggio del cerchio equivalente corrispondente all'isosista di intensità $I_{r}$ e $\gamma$ il coefficiente di assorbimento, valutato $3.989+1.344$ per l'Italia Centrale. (Marcelli, Spadea, 1981, in press). 
dall'Osservatorio di Monte Porzio Catone dell'Istituto Nazionale di Geofisica:

$$
\begin{aligned}
& \varphi=42^{\circ} .730 \mathrm{~N} \quad \lambda=12^{\circ} .956 h=6 \text { (assegnato) } M_{\mathrm{L}}=5.5 \\
& H=21^{\mathrm{h}} 35^{\mathrm{m}} 36.9^{\circ}(\mathrm{GMT}) .
\end{aligned}
$$

\section{7 - AtTENUAZIONE}

Come già notato in precedenza l'andamento delle isosisme presenta un marcato allungamento lungo la direttrice N-S, prevalente verso $\mathrm{N}$, mentre lungo la direzione normale presenta uno schiacciamento verso $\mathrm{E}$. La figura 3 rappresenta questi andamenti;

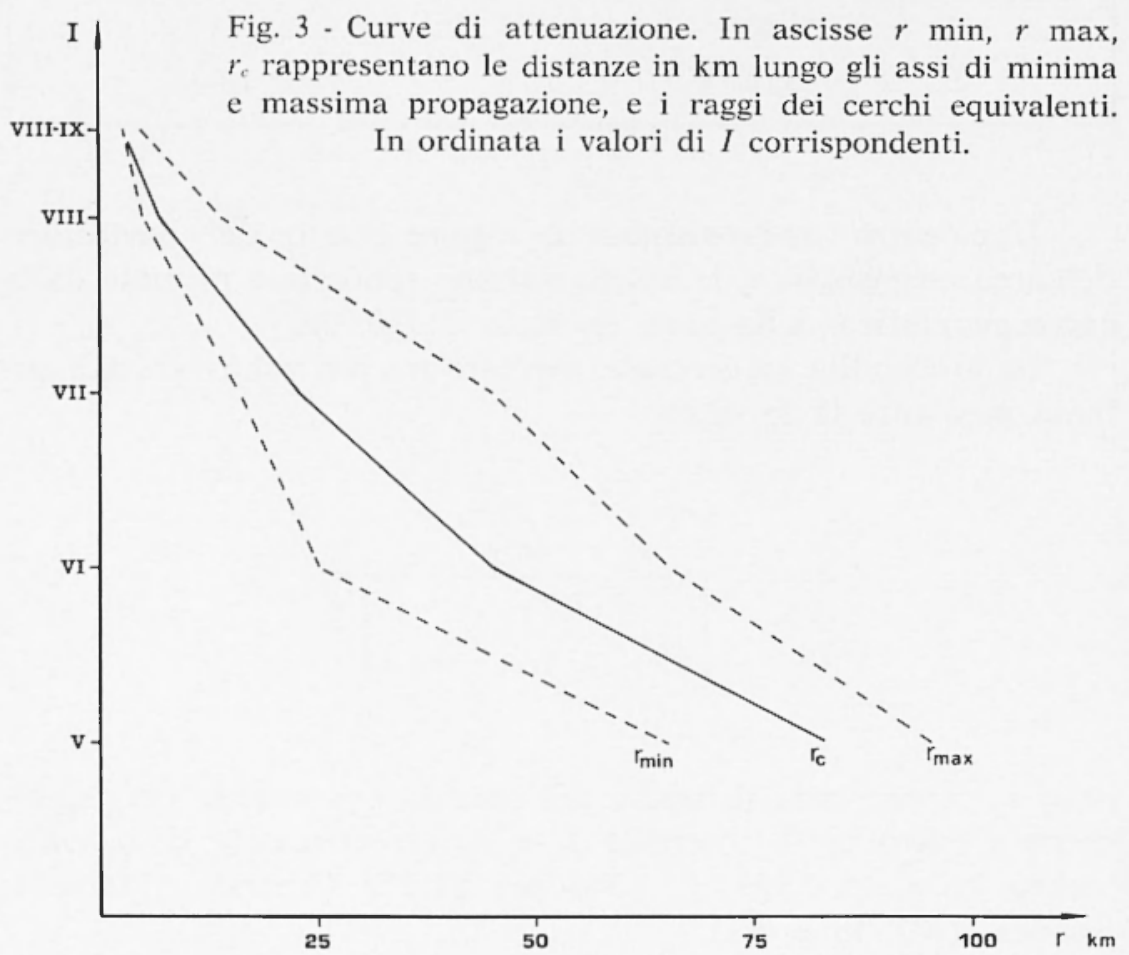


in ascissa sono riportati le misure in $\mathrm{km}$ lungo la direzione di massima e di minima propagazione, nonché quelle dei raggi dei cerchi equivalenti $\left(r_{c}\right)$; in ordinata i valori della intensità corrispondenti.

Sono stati calcolati i coefficienti di attenuazione $(\alpha)$ lungo le prevalenti direzioni del campo macrosismico mediante la relazione:

$$
\alpha=\frac{2.30}{D_{\mathrm{c}}-H} \cdot \quad \log \frac{I_{o}}{I_{c}}
$$

dove $D_{c}$ è la distanza ipocentrale ed $I_{0}$ l'intensità epicentrale. Nella tab. 5 sono riportati i coefficienti di attenuazione per le

T A B E L L A 5

Coefficienti di attenuazione

\begin{tabular}{|c|c|c|c|}
\hline \multirow{2}{*}{ Direttrice } & $D_{c}$ & $\alpha$ & $x$ per VIII $<I_{c}<$ VI \\
\cline { 1 - 2 } N VIII & 17 & 0.00841 & $0.00667+/-0.00093$ \\
VII & 46 & 0.00537 & \\
VI & 66 & 0.00624 & \\
V & 95 & 0.00620 & \\
E VIII & 11 & 0.05130 & $0.03124+/-0.00867$ \\
VII & 19 & 0.02187 & \\
VI & 27 & 0.02056 & \\
V & 66 & 0.00950 & \\
S VIII & 12 & 0.02744 & $0.01846+/-0.00446$ \\
VII & 21 & 0.01831 & \\
VI & 46 & 0.00964 & \\
V & 90 & 0.00658 & \\
W VIII & 12 & 0.02744 & $0.01736+/-0.00404$ \\
VII & 27 & 0.01146 & \\
VI & 36 & 0.01318 & \\
V & 65 & 0.00968 & \\
\hline
\end{tabular}


intensità dall'VIII al $\mathrm{V}$ grado secondo le quattro direttrici $\mathrm{N}$. E, S, W.

Di tutti questi coefficienti, gli AA. hanno ritenuto di fare la media limitandosi al VI grado in quanto l'attenuazione riscontrabile nell'isosista di V grado, se inserita nel calcolo della media, ne avrebbe prodotto un appiattimento. Dall'esame delle medie si evidenzia in maniera quantitativa quanto già osservato in maniera qualitativa, e cioè che la propagazione è nettamente favorita verso Nord $(\alpha=0,00667)$, e notevolmente sfavorita verso Est $(\alpha=0,003124)$.

Nelle altre due direzioni l'attenuazione è causa di una propagazione più uniforme.

\section{8 - Precedenti Storici}

L'esame degli eventi storici dell'Umbria ha messo in evidenza l'elevato grado di sismicità di tutta la Valnerina ed in particolare della zona di Cascia e Norcia. Nella tab. 6 sono riportati gli eventi più rilevanti che hanno interessato i due Comuni. In particolare nell'ultimo secolo, sono avvenuti numerosissimi terremoti d'intensità tra "deboli " e "forti ", numerosi "molto forti ", e alcuni "fortissimi".

\section{9 - ConClusione}

Il rilevamento condotto dagli AA. e tutti gli altri dati raccolti hanno permesso di definire compiutamente l'andamento del campo macrosismico ed i suoi parametri. Confrontando i risultati ottenuti con i dati microsismici è possibile notare una buona corrispondenza nel valore della profondità ipocentrale che rivela le caratteristiche superficiali dell'evento. Per quanto riguarda invece la posizione dei due epicentri macro e microsismico si può constatare una diversità nelle due determinazioni. Questo probabilmente perché il calcolo dei parametri epicentrali della scossa principale è stato fat to utilizzando i dati delle stazioni della Rete Sismica Nazionale senza il necessario ausilio di quelle 
locali. Infatti successivamente, entrando in funzione una rete locale, si è avuto un netto addensamento degli epicentri a sud di Norcia, all'interno dell'isosisma di VIII grado, in un'area molto prossima a quella megasismica. Evidenti, seppur limitati, gli effetti locali, sia positivi che negativi. Fra quelli positivi un cenno

TABELla 6

Eventi storici

\begin{tabular}{|c|c|c|c|c|c|}
\hline DATA & & 0 & $\lambda$ & $I_{c}$ & LOCALITA' \\
\hline 1 DICEMBRE & 1328 & 42.833 & 12.917 & IX & NORCIA \\
\hline 27 AGOSTO & 1567 & 42.750 & 13.000 & VII & NORCIA \\
\hline 5 NOVEMBRE & 1599 & 42.667 & 13.000 & VIII & CASCIA \\
\hline 10 GENNAIO & 1600 & 42.667 & 13.000 & VII & CASCIA \\
\hline 14 GENNAIO & 1703 & 42.750 & 13.083 & $\mathrm{x}$ & NORCIA \\
\hline 25 FEBBRAIO & 1703 & 42.500 & 13.000 & VIII & NORCIA \\
\hline 8 APRILE & 1703 & 42.530 & 13.000 & VII & CASCIA \\
\hline 4 OTTOBRE & 1716 & 42.750 & 13.000 & VII & CASCIA \\
\hline 27 GIUGNO & 1719 & 42.500 & 13.000 & VIII & NORCIA \\
\hline GENNAIO & 1760 & 42.500 & 13.000 & VII & CASCIA \\
\hline 3 SETTEMBRE & 1812 & 42.750 & 13.000 & VIII & NORCIA \\
\hline 22 AGOSTO & 1859 & 42.833 & 13.000 & VIII & NORCIA \\
\hline 27 MAGGIO & 1860 & 42.833 & 13.600 & VIII & NORCIA \\
\hline 23 FEBBRAIO & 1879 & 42.783 & 13.050 & VII-VIII & NORCIA \\
\hline 2 AGOSTO & 1964 & 42.700 & 13.000 & VII & CASCIA \\
\hline 4 OTTOBRE & 1971 & 42.800 & 13.200 & VII & NORCIA \\
\hline
\end{tabular}

particolare va fatto per quei paesi che sono compresi nell'area di massimo scuotimento (Castel S. Maria, Chiavano, Civita, Trimezzo); altri esempi a Giappiedi (Fraz. di Norcia) a Montebufo e Villa Campanara (Fraz. di Preci).

Effetti locali negativi, invece, sono stati riscontrati a Castelluccio, Nottoria e comunque in tutta l'area dei Monti Sibillini. 
Le possibili cause vanno ricercate nell'interazione fra i tipi litologici su cui poggiano le strutture e le caratteristiche delle stesse.

Infatti, tutte queste località poggiano su colli di natura calcarea che, data la superficialità dell'ipocentro, hanno trasmesso per intero le sollecitazioni senza alcuno smorzamento nel contenuto in frequenze, facendo entrare in risonanza le costruzioni tipiche della zona, caratterizzate da bassi periodi propri.

A questo va aggiunta la scadente qualità della maggior parte delle strutture in muratura (in genere non cordolate) che utilizzano pietre non squadrate o addirittura tondeggianti legate con malte molto povere, con tetti spingenti e fondazioni assenti o poco profonde.

Quindi l'andamento generale della propagazione macrosismica tende a sovrapporsi all'assetto strutturale dell'area in esame; come se la propagazione dell'energia sismica lungo la superficie, venisse favorita nel seguire le strutture tettoniche e ne trovasse un impedimento in direzione trasversale. In questa ipotesi potrebbe trovare una possibile spiegazione la maggiore attenuazione riscontrata verso Est.

Questo lavoro segna la ripresa da parte dell'I.N.G., nonostante le difficoltà incontrate, dello studio macrosismico sistematico di campagna, per eventi disastrosi interessanti la penisola italiana.

\section{RINGRAZIAMENTI}

Si ringrazia il Comando Generale dell'Arma dei CC. per il valido contributo apportato con l'invio e la compilazione di oltre 500 questionari, e il Centro di Coordinamento di Norcia per la disponibilità dimostrata. 
numero di codice ING

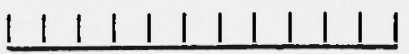

\title{
ISTITUTO NAZIONALE DI GEOFISICA
}

\section{ROM A}

\section{Sezione Macrosismica}

\author{
QUESTIONARIO
}

Richiesta di notizie sul terremoto

del giorno.

Si prega di restituire, nel più breve tempo possibile, il questionario compilato barrando con una croce la casella, a destra, $\mid$ si $|,| \overline{n o} \mid$, non so , corrispondente alla risposta che si vuole dare, e sottolineando - quando possibile l'effetto osservato.

Nel caso di più scosse di terremoto, compilare un questionario per ogni scossa di terremoto.

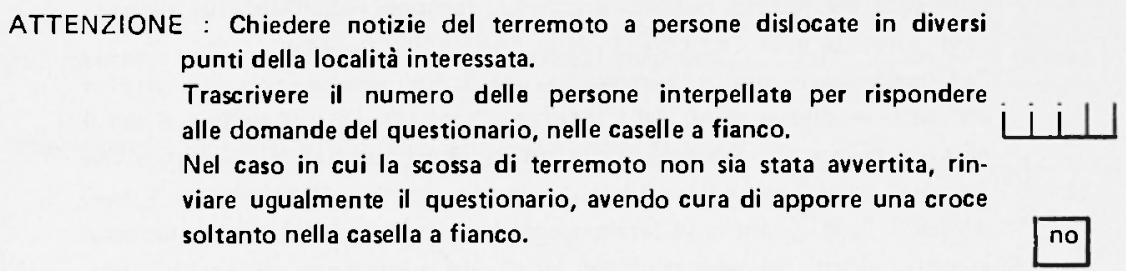

Nome della località

Regione

II terremo to è stato avvertito il

alle ore $\bigsqcup \perp$ (ora solare)

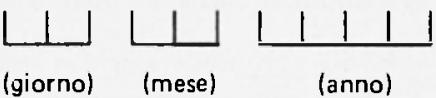

(ore) (minuti)

AVVERTENZE: Le colonne a sinistra delle domande e le note fra parentesi di seguito alle domande, non interessano il compilatore del questionario, ma l'Istituto competente.

a) - Trascrivere i giorni da 01 a 31, occupando le due caselle.

b) - Trascrivere i mesi da 01 a 12, occupando le due caselle.

c). Trascrivere l'anno per esteso, occupando le quattro caselle.

d) - Trascrivere le ore da 00 a 24, occupando le due caselle.

e) - Trascrivere i minuti da 00 a 59, occupando le due caselle.

f) - Nei mesi in cui è in vigore l'ora legale, trascrivere l'ora solare, ricordandosi di togliere un'ora.

All. 1 - Questionario macrosismico elaborato dall'Istituto Nazionale di Geofisica. 


\title{
CHIARIMENTI PER IL COMPILATORE
}

\author{
DEL OUESTIONARIO
}

1) Gli aggettivi poche, molte, moltissime, tutte si riferiscono al numero di persone che, al momento del terremoto, si trovavano in ambienti chiusi o all'aperto.

2) Nel questionario si è cercato di riassumere, sotto forma di domande, alle quali rispondere con un si, un no, un non so, tut to ciò che le persone hanno percepito e osservato direttamente prima o durante il terremoto; cioè le conseguenze immediate del terremoto sulle persone, sulle cose all'interno degli edifici (case, scuole, chiese, ospedali, bar, luoghi di riunione, ecc.). sull'ambiente circostante (terreno, corsi d'acqua, strade, montagne, ecc.). sulle strutture (edifici pubblici e privati), vengono rispettivamente raggruppate sotto la voce "EFFETTI DEL TERREMOTO SU

Per questa ragione è indispensabile che il compilatore interpelli persone dislocate in diversi punti della località interessata dal terremoto, e che il numero di persone interrogate sia sufficientemente grande. Soltanto una indagine accuratamente svolta dalle persone incaricate di compilare il questionario, può garantire il servizio, collaborando con i ricercatori alla soluzione di alcuni dei tanti problemi legati alla prevenzione dal rischio sismico e alla protezione del territorio.

3) Le costruzioni sono contraddistinte dalle lettere $A ; B$; $C$ in relazione al TIPO di costruzione (materiali usati, e periodo in cui sono state costruite):

TIPO A: edifici o strutture vecchi o mal costruiti (in mattoni cotti o in creta o in pietrame di campol

TIPO B: edifici a strutture in materiali comuni e più recenti lmattoni comuni. case a metà legno metà pietra, in pietra naturale tagliata).

TIPOC: edifici o strutture nuove in materiale consolidato (costruzioni in cemento armato. in leano ben costruite, antisismiche, prefabbricati in cemento armato e legno, armati in resina ecc.).

Se nella località interessata dal terremoto, è possibile reperire anche solo alcune delle informazioni 3), il compilatore può sottolineare il TIPO di costruzione, di interesse sostanziale per una elaborazione ottimale del questionario.

4) Se nella domanda sono descritti più effetti e ne è stato osservato anche uno soltanto, sottolinearlo. La risposta è ugualmente importante. 


\section{EFFETTI DEL TERREMOTO SULLE PERSONE}

Le persone che hanno avvertito il terremoto all'interno di case, scuole, chiese, ospedali, cinema, luoghi di riunione ecc., sono state:

(03-mcs) poche, sedute o a letto, che non si sono rese subito conto che si trattava di terremoto

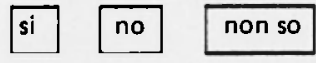

(03-mcs) poche, in movimento, che non si sono rese subito conto che si trattava di terremoto...

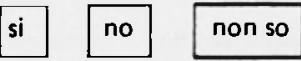

(04-mcs)

Molte, senza spavento

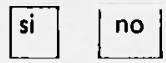

non so

(05-mcs-msk) tutte di giorno; molte di notte si svegliano....

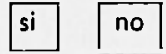

non so

(06-mcs-msk) tutte, molte delle quali spaventate fuggono all'aperto

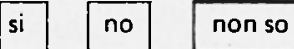

$(07 \cdot \mathrm{msk})$ tutte, moltissime delle quali fuggono all'aperto

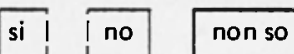

Le persone che hanno avvertito il terrumoto all'aperto, strade. piazze, giardini, ecc., sono state:

(04-mcs)

poche
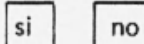

non so

(05-mcs-msk)

moltissime con spavento
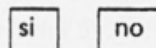

non so

(06-mcs-msk)

tutte con spavento 


\title{
EFFETTI DEL TERREMOTO SULLE COSE ALL'INTERNO DI EDIFICI PUBBLICI E PRIVATI
}

\author{
E' stato osservato:
}

(03-msk) Leggera oscillazione di oggetti sospesi, osservata soltanto negli ultimi piani delle case

$\mid$ si ||$\overline{n o} \mid$ non so

(04-mcs-msk) Leggera oscillazione di oggetti sospesi; di liquidi nei recipienti colmi

$|\overline{s i}||\overline{n o}|$ non so

(05-mcs-msk) Forte oscillazione di oggetti sospesi; di liquidi in recipienti colmi con piccoli versa. menti; qualche orologio a pendolo si è fermato; i quadri appesi si sono mossi o hanno sbattuto contro la parete

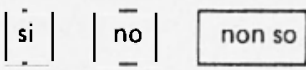

(06-mcs-msk) Fortissima oscillazione di oggetti sospesi; di liquidi in recipienti colmi con traboccamento

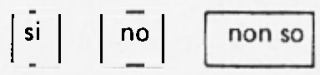

(05-mcs-msk) Suono di piccole campanelle sospese.

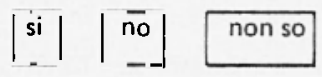

(06-mcs-msk) Suono di piccole campane nei campanili delle Chiese, nelle scuole ecc.

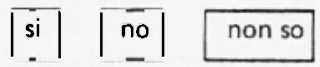

(07-mcs-msk) Suono di grandi campane nei campanili delle Torri o delle Chiese

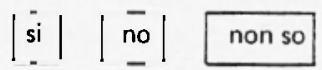

(04-mcs-msk) Leggero scuotimento di porte, finestre, suppellettili; vibrazione di pareti e pavimenti; tintinnio di vetri di finestre o di vetrine di mobili

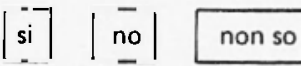


(05-mcs-msk) Porte, finestre, sportelli hanno sbattuto o si sono spalancati

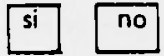

non so

(05-mcs-msk) Scricchiolio di mobili, di travi nei soffitti....

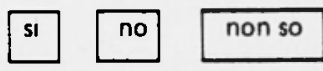

(06-mcs-msk) Spostamento o caduta di qualche mobile leggero; caduta di quadri appesi alle pareti....

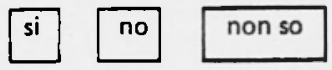

(08-mcs-msk) Spostamento o caduta di qualche mobile pesante e solido.

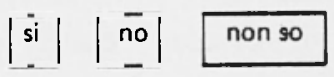

(09-msk) Violento spostamento e caduta di mobili, con conseguenti spaccature

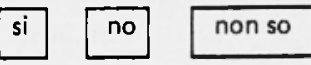

(05-mcs-msk) Spostamento o caduta di piccoli soprammobili

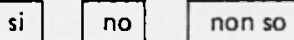

(06-mcs-msk) Rottura di piatti e bicchieri per urto e caduta; caduta di qualche libro dalle mensole.......

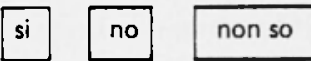

(07)-mcs) Caduta e rottura di oggetti pesanti, da mensole o tavoli 


\section{EFFETTI DEL TERREMOTO SULL'AMBIENTE CIRCOSTANTE}

(montagne, terreno, campi, strade, corsi d'acqua, canali, pozzi, ecc.)

\section{E' stato osservato:}

(06-msk)

Qualche spaccatura in terreni umidi

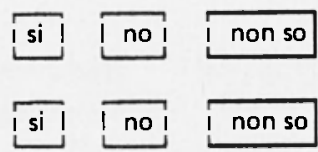

(07-mes-msk)

Qualche spaccatura nelle strade sterrate .......

(08-mcs-msk) Molte spaccature e crepe in terreni umidi o in pendio

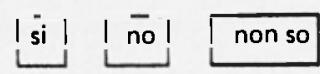

(09-msk)

Larghe spaccature negli argini dei fiumi; piccole spaccature in strade pavimentate o asfaltate

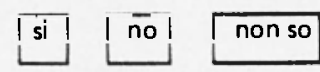

(10-mcs-msk) Crepe lunghe e profonde con avvallamenti in strade pavimentate $o$ asfaltate

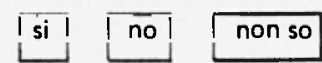

(06-msk)

Qualche frana sulle montagne vicine

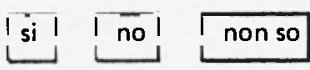

(07-mcs) Strade in pendio e terreni sabbiosi o ghiaiosi franano in alcuni punti

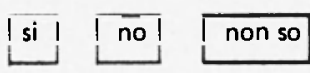

(09-msk) Molte frane dalle montagne con caduta di massi rocciosi

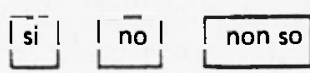

(11-mcs-msk) Frane numerosissime con smottamenti e sconvolgimenti del terreno

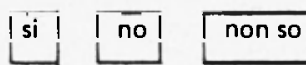

(07-mcs-msk) Intorbidamento di stagni, canali d'irrigazione, corsi d'acqua, causato dall'agitazione dell'acqua; cambiamento di livello nell'acqua dei pozzi

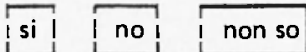


(08-mcs-msk) Intorbidamento dei corsi d'acqua con trasporto di fango e sabbia

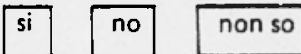

(09-msk) Molti pozzi si sono asciugati; in molti pozzi asciutti è tornata acqua.

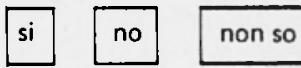

(10-msk) Straripamento di fiumi, canali, laghi a causa di grandi frane o smottamenti di terreno, o per alte ondate

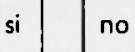

non so

(08-msk)

Alcuni alberi si sono piegati o spezzati

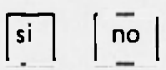

non so

(12-mcs-msk) Crollo di montagne; apertura di voragini; isole emerse o inghiottite; l'ambiente circostante è totalmente sconvolto

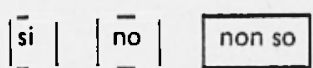




\title{
EFFETTI DEL TERREMOTO SULLE COSTRUZIONI E SULLE STRUTTURE
}

\author{
$E^{\prime}$ stato osservato:
}

(05-msk) Caduta di calcinacci o piccoli pezzi di intonaco in case vecchie $\mathrm{e}$ mal costruite, Tipo A (5\% 1A)

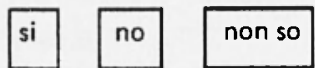

(06-mcs-msk) Molte lesioni leggere in case vecchie e mal costruite, Tipo A; qualche crepa con caduta di intonaco in costruzioni di Tipo B (50\% 4A-5\%2A-5\% 1B).

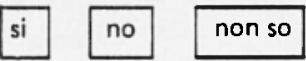

(07-mcs-msk) Crolli parziali e lesioni in alcune case vecchie e mal costruite, Tipo $A$; lievi danni e piccole lesioni in case di Tipo $C(50 \% 3 A$ $5 \% 4 A-50 \%$ iC)

si no non so

(08-mcs-msk) Crolli parziali con molte distruzioni nel $25 \%$ di tutti i tipi di edifici, Tipo A, B, C; qualche costruzione in legno o muratura e le. gno è stata spostata o spazzata via, Tipo $C$ $150 \% \quad 4 A-5 \%$ 5A-50\% 3B-5\% 4B-50\% 2C-5\% $3 C)$

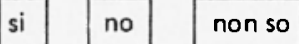

(09-mcs-msk) Crollo totale del 50\% degli edifici vecchi e mal costruiti, Tipo A; crolli parziali e danni gravissimi agli edifici di Tipo B e C $150 \%$ $5 A-50 \% 4 B-5 \%$ 5B-50\% 3C-5\% 4C)

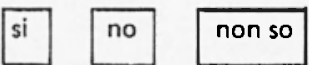

(10-mcs-msk) in generale gravi danni con crolli nel 75\% di tutti gli edifici; crolli totali in case vecchie e mal costruite, Tipo A; distruzioni e gravissimi danni agli edifici di Tipo $B$ eC. (75\%5A; 50\%5B-50\%4C-5\%5C) 
(11-mcs-msk) Crollo totale di quasi tutti gli edifici di Tipo A, B, C (100\%5A-75\%5B-75\%4C).

(06-mcs)

Caduta dai tetti di qualche comignolo e qualche tegola

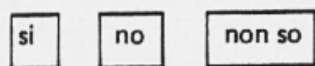

(07-mcs-msk) Caduta o rottura di molti comignoli e tegoli: con slittamento di essi sui tetti (50\%2B).

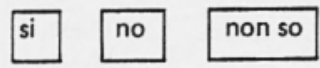

(08-mcs) Crolli parziali di campanili, ciminiere e muri di cinta

\section{no \\ non so}

(08-mcs-msk) Caduta di qualche statua o scultura dal piedistallo; spostamento di lapidi;ribaltamento di pietre tombali

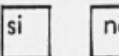

non so

(09-msk)

Caduta di monumenti e colonne

(07-msk)

Danni alle saldature delle tubazioni di acqua e/o di gas

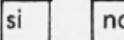

non so

(08-msk)

Qualche crepa nelle tubazioni sotterranee di acqua e/o di gas

si

no

non so

(10-mcs-msk) Piegamenti e rotture nelle tubazioni sotterranee di acqua e/o di gas

\section{si}

no

non so

(11-mcs-msk) Distruzione totale delle tubazioni di acqua e/o di gas

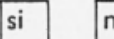

non so

(10-mcs-msk) Danni pericolosi per la stabilità di ponti e dighe

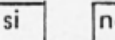

non so

(11-mcs-msk) Crollo totale di ponti con pilastri, viadotti, superstrade; danni gravissimi alle dighe 
(10-mcs-msk) Leggero piegamento delle rotaie dei tram e dei treni

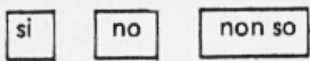

(11-mcs-msk) Danni gravissimi alle rotaie dei tram e dei treni, con caduta delle linee aeree

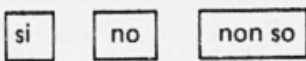

(12-mcs-msk) Distruzione totale delle costruzioni e di tutte le strutture sopra e sotto la superficie terrestre $(100 \% 5 \mathrm{~A} ; 100 \% 5 \mathrm{~B} ; 100 \%$ 5C)

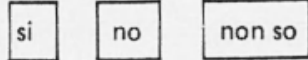

\section{RICHIESTA DI INFORMAZIONI}

a) - Numero di edifici privati o pubblici relativo all'ultimo censimento

b) - Numero di edifici inabitabili per i danni provocati dal terremoto

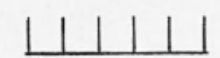

c) - Numero di edifici lesionati

d) - Quanti edifici vecchi hanno subito danni?

e) - Quanti edifici vecchi ristrutturati hanno subito danni?

f) - Quanti edifici costruiti prima del 1900 e il 1940 hanno subito danni?

g) - Quanti edifici costruiti dopo l'ultima guerra (1945.1971) hanno subito danni?
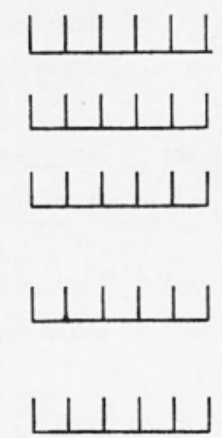

h) - Quanti edifici con strutture in cemento armato hanno subito danni?

i) - Quanti abitanti conta la località?

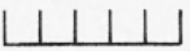




\section{EFFETTI OSSERVATI PRIMA, CONTEMPORANEAMENTE, O DOPO IL TERREMOTO}

a) - Rombo sotterraneo, rimbombo, rumore di vento, brontolii, rumore simile ad esplosione (Breve descrizione:

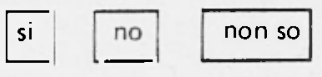

b) - Effetti luminosi

c) - Agitazione negli animali

(Breve descrizione del comportamento degli animali sta. bilendo, quando è possibile, quanto tempo prima del terremoto è stato osservato:

d) - Riscaldamento dell'acqua in pozzi o cisterne

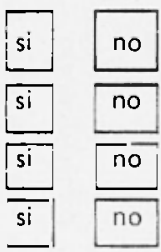

e) - La popolazione si è spaventata?

f) - La popolazione sì è riversata all'aperto?

g) - Tutta la popolazione è stata presa dal panico?

h) - Quante persone hanno lasciato la località dopo il terremoto?

i) - OSSERVAZIONI PERSONALI: 
UFFICIO CENTRALE DI ECOLOGIA AGRARIA E OIFESA DELLE PIANTE COLTIVATE DALLE AVVERSITÁ METEORICHE - ROMA SERVIZIO SISMICO

Nod. $S_{z} \cdot T 1$ LCFA

Francalura a carico del destinatario da addebilarsi sul conto di credilo n. 484 presso I.Ufficio di Roma Cenlro (Aulorizn. $1, j 4025,322$ del $\left.6, X_{11}, 1961\right)$

\title{
All' 'Ufficio Centrale di Ecologia Agraria \\ e Difesa delle Piante Coltivate dalle Aversitia Meterichte
}

\author{
00186 - ROMA ${ }^{(1)}$ \\ Via del Caravita N. 7-a
}

Si raccomanda di usare una cartolina separata per ogni scossa distinta a meno che si tratti di scosse a pochi secondi d'intervallo tra loro.

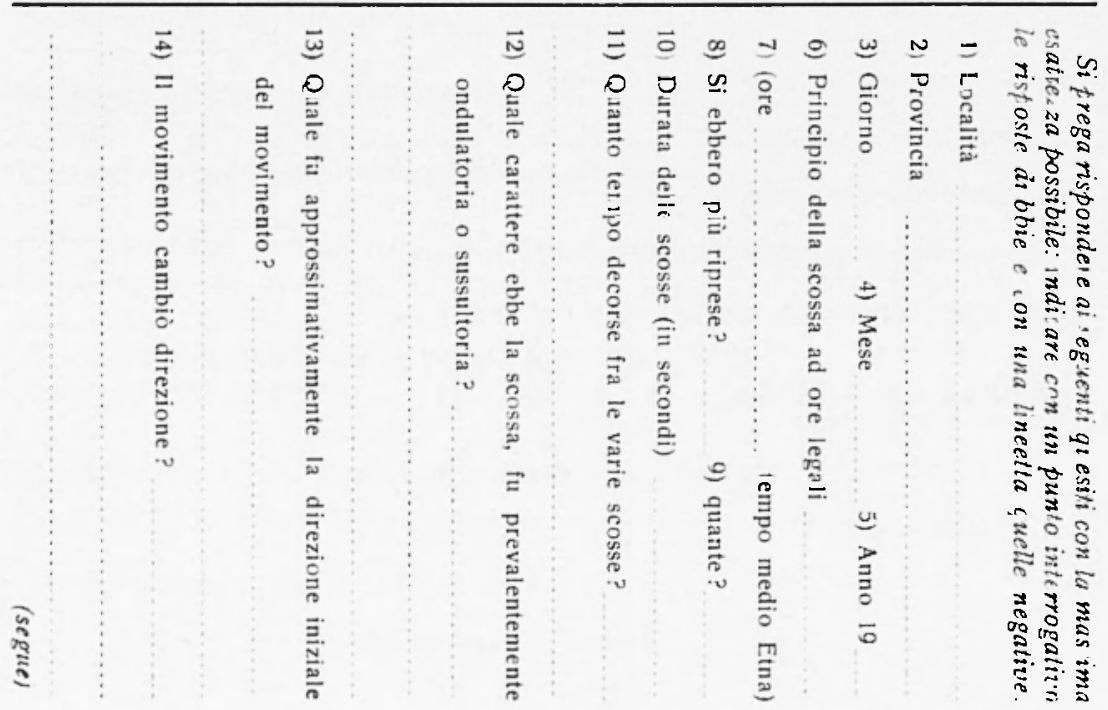

All. 2 - Cartolina macrosismica in uso fino al 1974. 
1.) Effetti delia scossa (dire se la scossa è stata avvertita da pocbi o da molti, reLativamenle al numero degli abitanti, e se coloro che l'avvertirono erano seduti, in cammino, al lavoro, in letto desti o addormentati, ccc. se abbia prodotto tremolio di piccoli o grandi oggetti, invetriate, porte, con o senza suono di rampanelli, fenditur. gravi o leggere in poche o molle case bene o male cosiruite: caduta totale o par ziale di edifici, vittime isolatc o numerose, ecc.) (Vedi scala sismica Mercalli)

16) Intensità (espressa in gradi della scala sismica Mercalli)

17) Segni negli animali (precedenti o contemporanei?)

18) Fenomeni luminosi

19) Rombi (sotterranei o nell'aria? precedenti, contemporanei o susseguenti alla scossa ?)

20) Fenomeni presentati dal mare, dai laghi, fiumi, pozzi d'acqua o sorgenti

21) Variazioni della temperatura di acque termali, fumarole, salse, ecc.

\section{2) Note diverse}

Spedita il

Nome e quallfloa del Relatore

Pervenuta il

registrata al $N$.

L'Addetto

V. II Capo Sezione 
ISTITUTO NAZIONALE DI GEOFISICA

Osservatorio dI MONTE PORZIO CATONE - ROMA

SCHEDA PER INDAGINE MACROSISMICA

Terremoto del 19. alle ore (solare)

REGIONE LOCALITA'

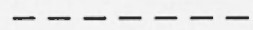

PERS ONE
QUANTITA'
poche
molte
quasi tutte
tutte
SENSAZIONI
risveglio
paura
sbandamento
vuoto
difficoltá nel movimento
impossibilitá di movimento
pánico e fuga all'apero

$\begin{array}{cc}\text { IN CASA } & \text { ALL'APERTO } \\ \square & \square \\ \square & \square \\ \square & \square \\ \square & \square\end{array}$

PERCEZIONI VISIVE

interruzione energia elettrica $\square \quad$ breve $\square$ lunga $\square$ fenomeni luminosi

\begin{tabular}{lcc}
\hline OSCILLAZIONE LAMPADARI & PIANO TERRA & PIANI SUPERIORI \\
piccola & $\square$ & $\vdots$ \\
grande & $\square$ & $\square$ \\
grandissima & $\square$ & $\square$
\end{tabular}

\begin{tabular}{lcccc}
\hline SUPPELLETTILI & SPOSTAMENTO & RIBALTAMENTO & CADUTA & ROTTURA \\
quadri appesi & $\square$ & $\square$ & $\square$ & \\
soprammobili leggeri & $\square$ & $\square$ & $\square$ & $\square$ \\
mensole o mobili leggeri & $\square$ & $\square$ & $\square$ & $\square$ \\
libri & $\square$ & $\square$ & $\square$ & $\square$ \\
oggetti pesanti & $\square$ & $\square$ &
\end{tabular}

\begin{tabular}{lcccc}
\hline PERCEZIONE AUDITIVA & PRIMA & CONTEMPOR. & DURANTE & DOPO \\
rombo & $\square$ & $\square$ & $\square$ & $\square$ \\
brontolio & $\square$ & $\square$ & $\square$ & $\square$ \\
colpo di vento & $\square$ & $\square$ & $\square$ & $\square$ \\
boato & $\square$ & $\square$ & $\square$ & $\square$ \\
esplosione & $\square$ & $\square$ & $\square$ & $\square$ \\
agitazione negli animali & $\square$ & $\square$ & $\square$ & $\square$ \\
VIBRAZIONI & & $\square$ & $\square$ & $\square$ \\
porte & $\square$ & $\square$ & $\square$ & $\square$ \\
finestre (vetri) & $\square$ & $\square$ & $\square$ & $\square$ \\
pareti & $\square$ & $\square$ & $\square$ & $\square$ \\
pavimenti & $\square$ & $\square$ & $\square$
\end{tabular}

All. 3 - Scheda per indagine macrosismica di campagna. 
SCRICCHIOLIO DI

pavimenti

travi di soffitti

mobili

\section{$\square$}

$\square$

\begin{tabular}{lcc}
\hline & SBATTERE DI & SPALANCARSI DI \\
porte & $\square$ & $\square$ \\
finestre sportelli di mobili & $\square$ & $\square$ \\
ante di armadi & $\square$ & $\square$ \\
quadri contro le pareti & $\square$ &
\end{tabular}

\section{SUONO DI}

campanelle sospese

allarmi

piccole campane

grandi campane

$\square$
$\square$
$\square$
$\square$

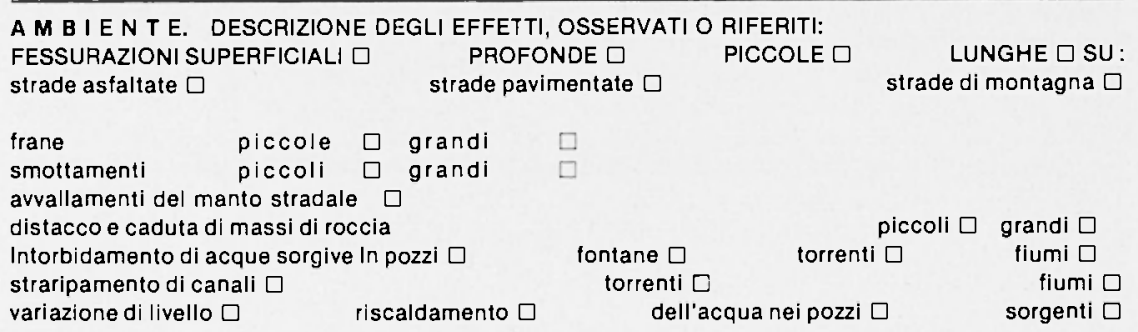

\section{COSTRUZIONI}

Descrizione danni su edifici in centrl agricoll, storici, recenti di tipo:

A) in pietra grezza, mattoni cotti legati con malte povere, vecchie mal ristrutturati con soffitti camorcar,ne o a travature in legno (scadenti)

B) In pletra squadrata, mattoni comuni, tufo, legati con buone malte o In legno con soffitti a travatura In ferro (mediocri)

C) in muratura cordolati, ben ristrutturati (quasi buoni)

D) case in legno berı progettate, in resine, prefabbricati in cemento armato, in legno e mattoni a struttura antisismica (buoni, ottimi)

caduta di calcinacci

piccoli pezzi di intonaco

pochi comignoli

poche tegole

grandi pezzi di intonaco

molti comignoli

molte tegole

slittamento di tegole sui tetti

rottura di tegole sul tetti

rottura di comignoli

$\begin{array}{llll}\text { A } & \text { B } & \text { C } & \text { D } \\ \square & \square & \square & \square \\ \square & \square & \square & \square \\ \square & \square & \square & \square \\ \square & \square & \square & \square \\ \square & \square & \square & \square \\ \square & \square & \square & \square \\ \square & \square & \square & \square \\ \square & \square & \square & \square \\ \square & \square & \square & \square \\ \square & \square & \square & \square\end{array}$

ROTAZIONE DI:

pinnacoli $\square$

statue sul piedistallo $\square$ 
CREPE

piccole e numerose superflciali lunghe e superficiali larghe e profonde trasversali

longltudinali

alla giunzione di pareti

a croce

su pllastri in c.a.
MURI INTERNI

A B C D

$\square \quad \square \quad \square \quad \square$

$\square \quad \square \quad \square \quad \square$

$\square \square \square \square$

$\square \square \square \square$

$\square \square \square \square$

$\square \quad \square \quad \square \quad \square$

$\square \square \square \square$
MURI ESTERN

A B C D

$\square \quad \square \quad \square \quad \square$

$\square \square \square \square$

$\square \quad \square \quad \square \quad \square$

$\square \square \square \square$

$\square \square \square \square$

$\square \quad \square \quad \square \quad \square$

$\square \square \square \square$
MURI MAESTRI

A B C D

$\square \quad \square \quad \square \quad \square$

$\square \square \square \quad \square$

$\square \square \square \square$

$\square \square \square \square$

$\square \square \square \square$

$\square \quad \square \quad \square \quad \square$

$\square \square \square \square$

Descrizione:

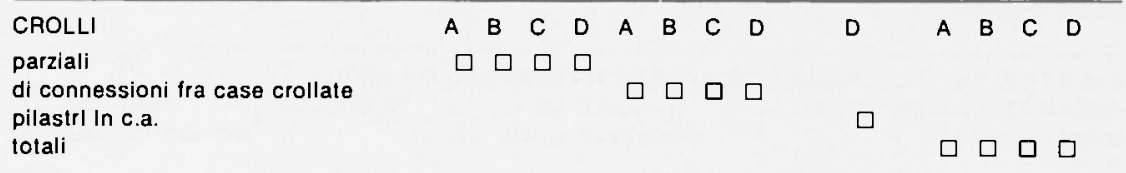

OSSERVAZIONI DEL RELATORE SU OUANTO OSSERVATO O RIFERITO PER DETERMINARE IL GRADO I (MCS.MSK): 


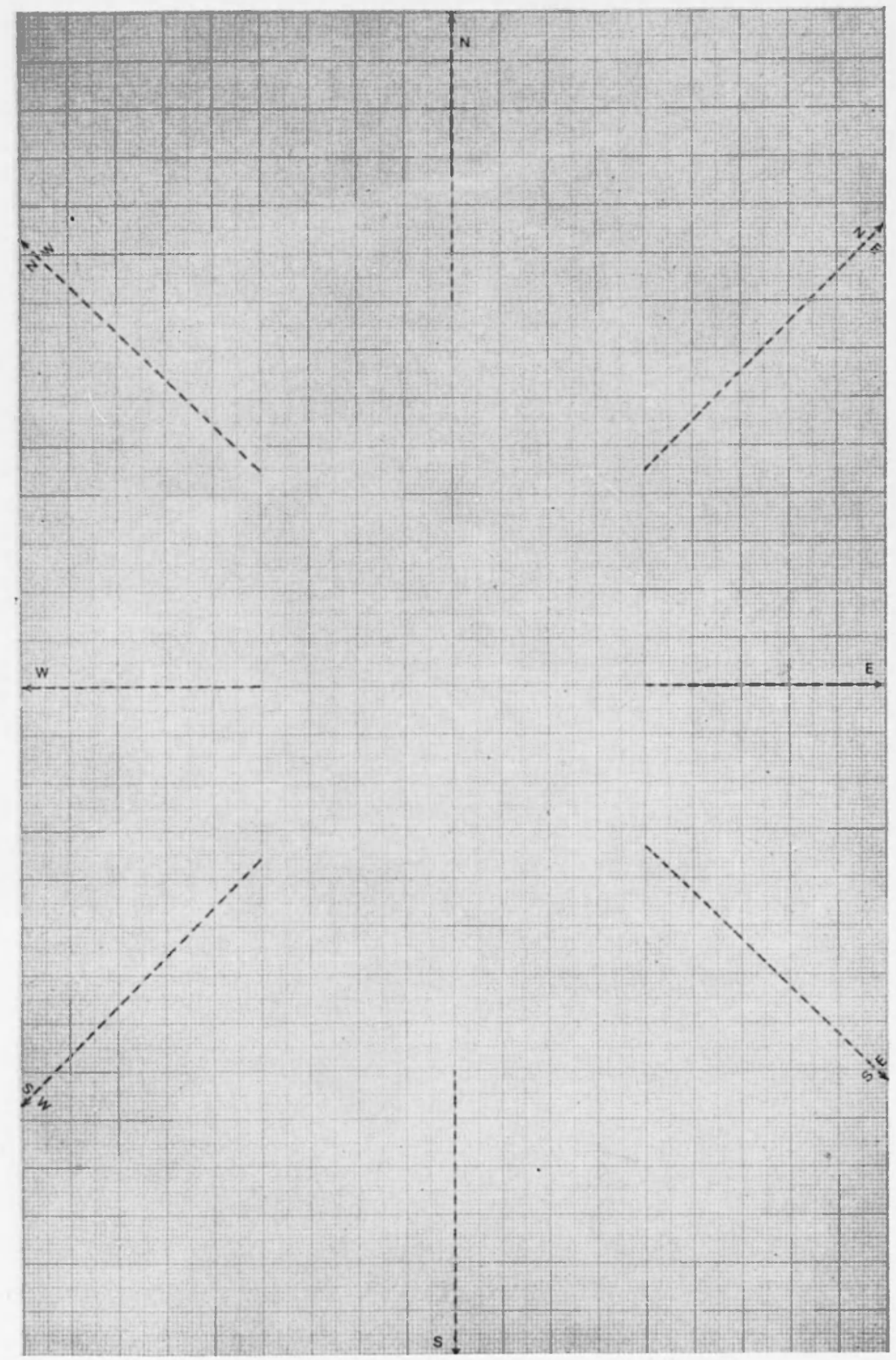




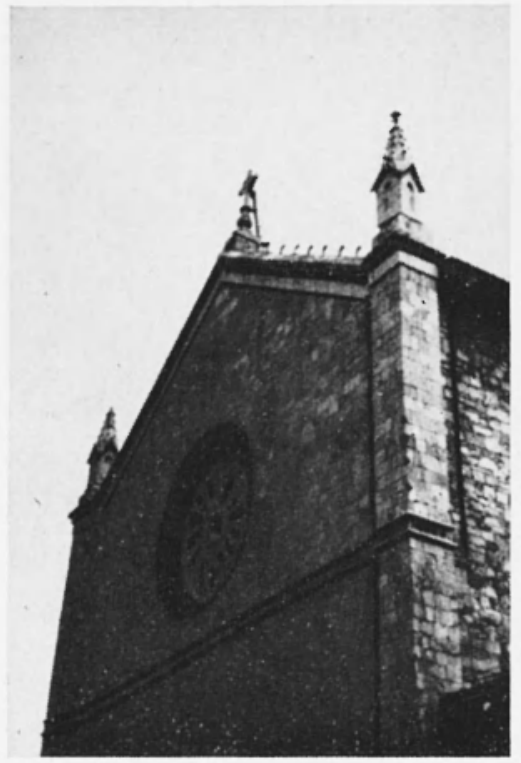

Foto 1 - Norcia, effetto di rotazione del pinnacolo della Chiesa.

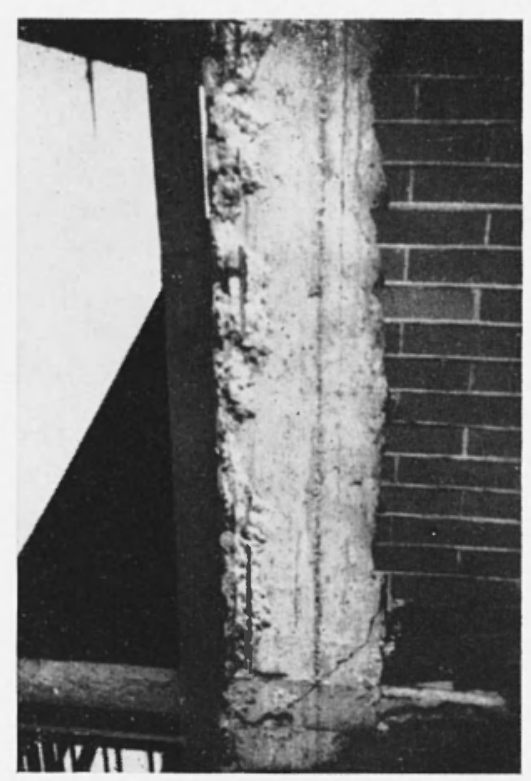

Foto 3 - Norcia, lesioni ad un pilastro di una struttura in cemento armato.

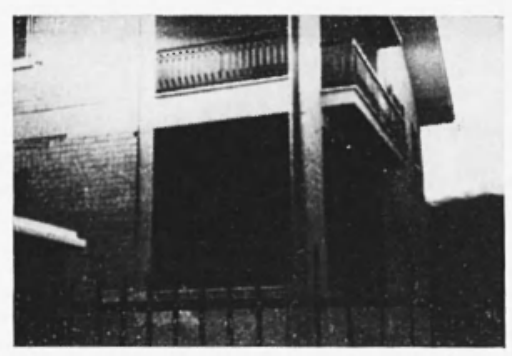

Foto 2 - Norcia, distacco di tamponature da una struttura in cemento armato.

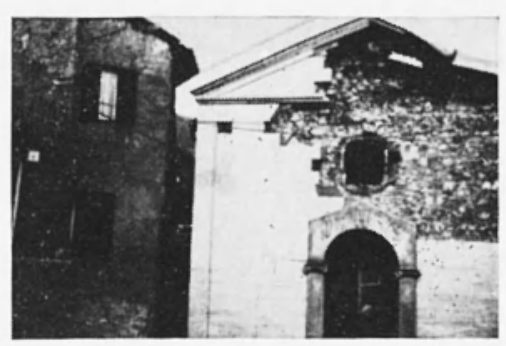

Foto 4 - Civita, crolli parziali della Chiesa. 


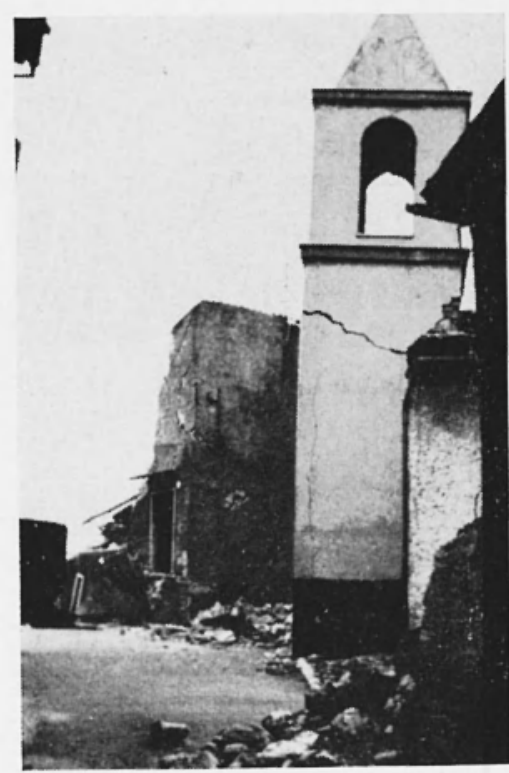

Foto 5 - Civita, crolli e gravi lesioni al Campanile della Chiesa.

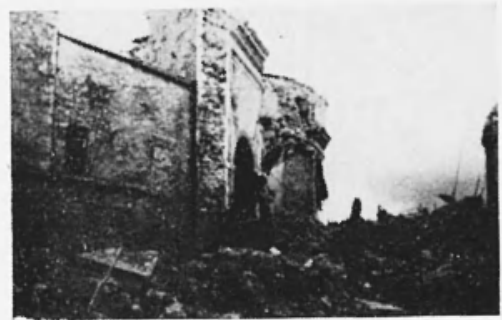

Foto 6 - Madonna della Neve.

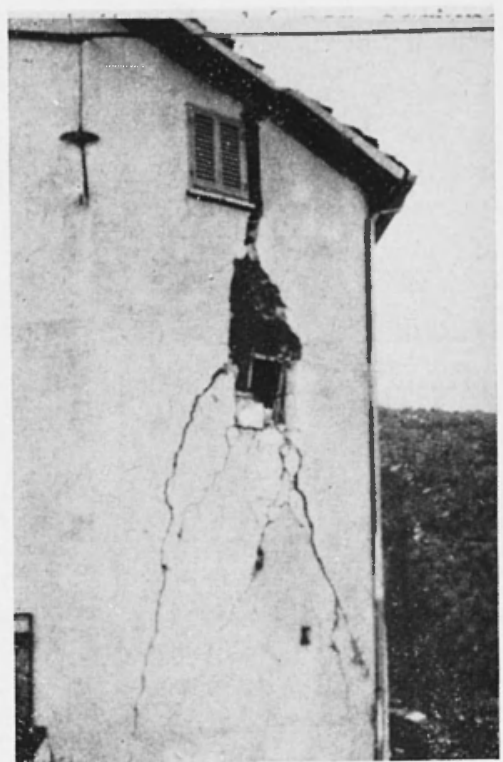

Foto 7 - Chiavano.

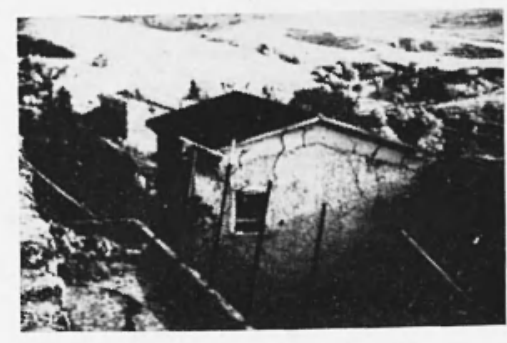

Foto 8 - Castel S. Maria, smottamenti. 


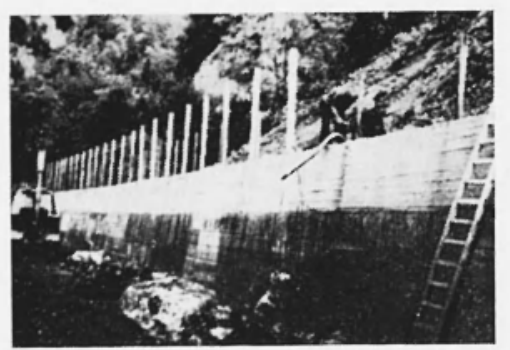

Foto 9 - Strada SerravalleNorcia, Frana.

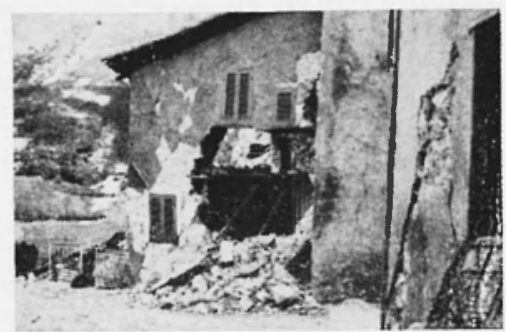

Foto 11 - Trimezzo.

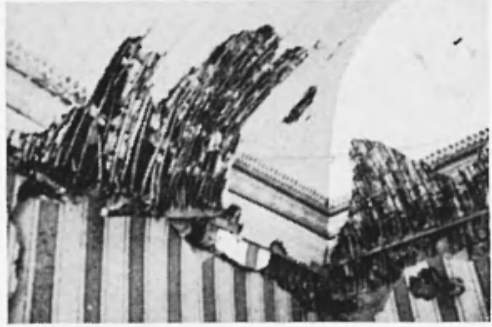

Foto 10 - Savelli, crollo parziale di un soffitio a camor. canne.

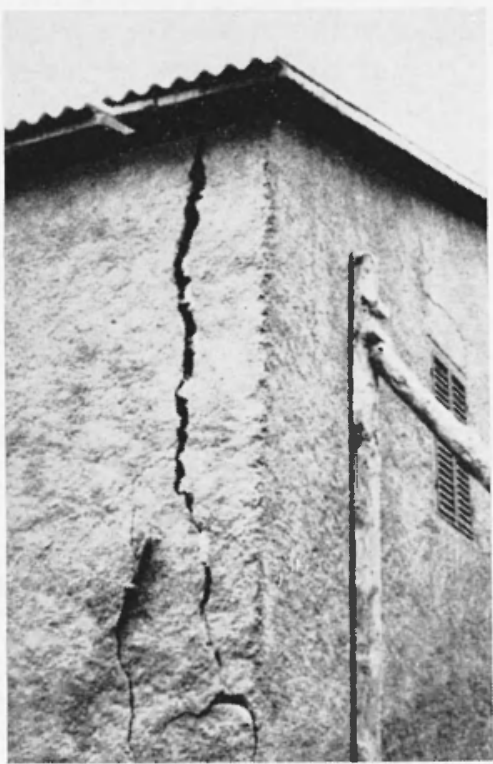

Folo 12 S. Marco. 
TABELLA 2

ELENCO DELLE LOCALITA' 
NIIM. LOCALITA

LAT. NORD LONG. EST INT.

REGIONE

CASTEL. S.MARIA

CHIAVANO

CIUITA

MAMONNA DEI LA NFUE

TRIMF ZZO

AUENOITA

CASCIA

CASTEI. S. GIOUANNI

COLLE S.STEFANO

COLI.E D' AUENDITA

COL.MUTINO

CORONEI LA

FOGL I ANO

FUSTAGNA

LF CASEINF

MAL.T I GNANO

MANIGI

NURCIA

OCRICCHIO

OPAIINA

PAI MAIUI $n$

PIETIRIPA

PUPOLI

PURO

SANTA TRINITA

SAVEI_L I

S. ANORFA

S. MARCO

TATZO

TROGNANI?

VILLA S.SILUESTRO

ATR I

COLFORCEI LA

OCOSLE

ONEI LI

PAGANELI I

PESCIA

SCIENI

S. ANATOLIA

VALCAI DARA

ARETO

ACCUNILLI

AGR I ANO

AT. IENA

ANCARANO

B ISEI.LI

BORGO CERRETO

BUDA

CAPUIOACIJUA

CASALI DI SERRAVA'LF.

CERFSOLA

CERRFTO DI SPOLFTO

CITTARFAI E

CIUITEILA

COLI AZZUNI

CORT IGL I ANO

FIANIO

FORSIVO

FRASCARO

GIAPP IEN I

LEGUGNE

LEONFSSA

LOGNA
4? $42 \quad 28$

423920

424820

424239

423841

$42 \quad 45 \quad 8$

42436

$4241 \quad$ B

$\begin{array}{lll}42 & 42 & 17\end{array}$

$\begin{array}{lll}42 & 45 & 27\end{array}$

424241

$\begin{array}{lll}42 & 38 & 13\end{array}$

424355

$4240 \quad 53$

42 39 8

424229

424246

$\begin{array}{llll}42 & 47 & 33\end{array}$

$4244 \quad 27$

423927

$42 \quad 43 \quad 31$

424426

42 454

4? 4356

424157

$42 \quad 4334$

42. 42 50

424248

$4243 \quad 18$

42 $37 \quad 45$

423859

$4243 \quad 38$

42446

4? 47 46

$42 \quad 4432$

424353

42415

42 4? 15

424423

4 ? 4424

4? $56 \quad 10$

424138

$42 \quad 4546$

424536

$4258 \quad 25$

$42 \quad 4731$

424851

$42 \quad 3759$

4 4 4111

$42473 \mathrm{~B}$

$42 \quad 46 \quad 19$

$4249 \quad 8$

$\begin{array}{lll}42 & 37 & 2\end{array}$

$4258 \quad 35$

425924

4?. $48 \quad 34$

$4249 \quad 28$

$\begin{array}{llll}42 & 47 & 55\end{array}$

4? 4948

$\begin{array}{llll}42 & 44 \quad 7\end{array}$

4 ? 48,

423358

424437 $\begin{array}{lll}13 & 7 & 1\end{array}$

VIII-IX

UTBRIA

$\begin{array}{lll}13 & 3 & 47\end{array}$

1375

13650

$13 \quad 7 \quad 17$

$13 \quad 259$

$13 \quad 046$

$13 \quad 343$

$\begin{array}{lll}13 & 3 & 43 \\ 13 & 3 & 46\end{array}$

13254

13446

$\begin{array}{lll}13 & 4 & 16 \\ 13 & 3 & 49\end{array}$

$13 \quad 249$

1343

1370

13328

135

13537

13545

13559

13227

$\begin{array}{lll}13 & 6 & 37\end{array}$

$\begin{array}{lll}13 & 6 & 17\end{array}$

1322

13428

13738

13727

13810

$\begin{array}{lll}13 & 3 & 15\end{array}$

13351

$13 \quad 31$ ?

13614

13,32

125953

13,58

13736

13925

13,48

13,36

13738

$\begin{array}{lll}13 & 3 & 47\end{array}$

139451

$13 \quad 158$

$\begin{array}{lll}13 & 2 & 24\end{array}$

13723

125925

125459

1335

$\begin{array}{lll}13 & 14 & 27\end{array}$

$\begin{array}{lll}13 & 34\end{array}$

125951

12 SS 1

13935

13,13

13 घ 24

125839

13355

13 D 53

$13 \quad 835$

125924

13136

125744

13043

VIII-IX UMBRIA

UIII-IX LAZIU

VIII UTBRIA

UIII

UIII

UIII

UIII

UIII

UII

UIII

UIII

UIII

UIII

VIII

UIII

UI II

UIII

UIII

UII

UIII

UII

UIII

VIII

UIII

UIII

UIII

UIII

UIII

UII-UII I

UII-UIII

UII-UIII

UII-UIII

VII-UIII

VII-UIII

VII-UIII

VII-UIII

VII-UI II

VII

UII

UI I

VII

VII

UII

VII

UII

UII

VII

VII

VII

VII

VII

UII

UII

UII

UII

UII

UII

VII

UII

UMBRIA

UMBR IA

UMBR IA

UMBRIA

UMBRIA

UMRRIA

UMBRIA

UTBRIA

UMBRIA

UMBR IA

UMBRIA

UMBRIA

UMBRIA

UMBRIA

UMBRIA

UTBR IA

UMBRIA

UMARIA

UMBRIA

UMBRIA

UMBRIA

UMBRIA

UMAR IA

UMBRIA

UMBRIA

UMBRIA

UMBR I A

UMBRIA

UTAR I A

UMBRIA

UMBRIA

UMBRIA

UMBRIA

UMBR IA

UMBR I A

LA7 IO

UMBRIA

UMBRIA 


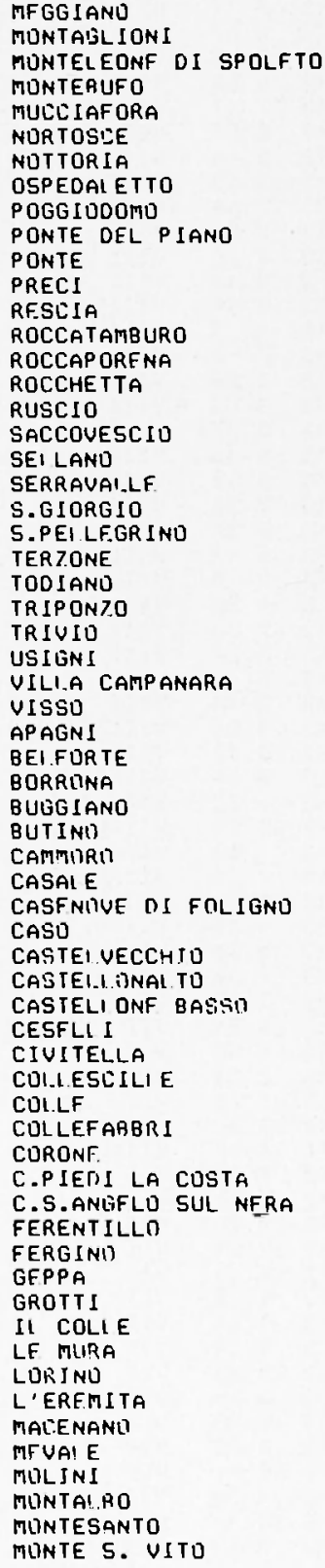

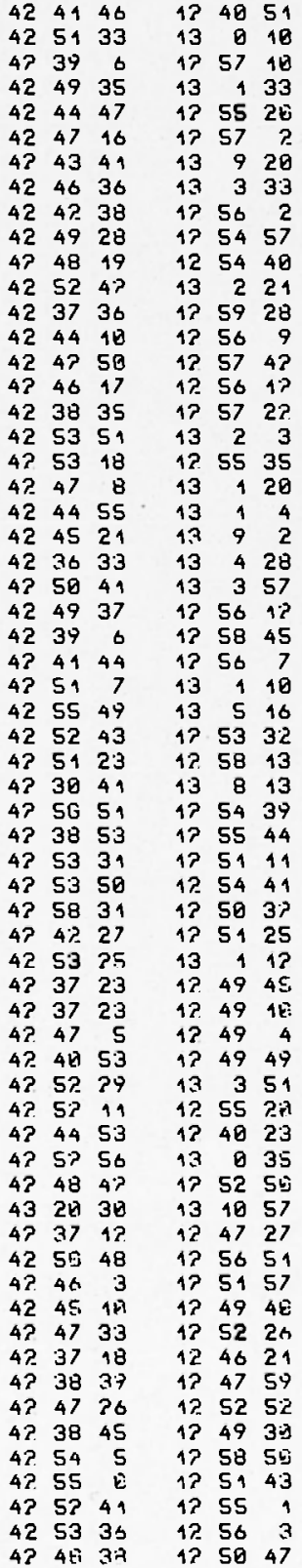

UI I

UMBR I A UTBR I A UTBR I A UTBR I A UTBRIA UTBRIA

UTBR I A

UTBRIA

UTBR IA

UTBR I A

UTBRIA

UTBR IA

UTBR I A

UITBR I $A$

UNBR I A

UTBR I A

UMAR I A

UTBRIA

UTBR I A

UTRR I $A$

UIMBR IA

UMBR I $A$

LAZ IO

UTBR I $A$

UTAR I $A$

UTBR I $A$

UTBR I $A$

UTRRIA

MARCHF

UTRR I A

UMBR I A

LA7 JO

UMBRIA

UTBR I A

UTRR I A

UNBRIA

UNBRIA

UTBR I A

UTRRIA

UTBRIA

UMBR I A

UTRRIA

UIRBR JA

UIRRIA

UTBR I A

UMRR I A

UMBR I A

UMBR I A

MARCHF.

UTRRIA

UIRRRIA

UTBR I $A$

UTBRIA

UMBRIA

UMRRI $A$

IITARI $A$

UTBR I A

UIRR I $A$

MARCHE

UMBR I A

UMBR I $A$

UMBR I A

UTBRIA 
VUM.

INT.

REGIONE

MUNTE CAVAL.LU

MUNTE FIORFL.LU

MONTE RIGOSO

MUCCIA

NICCIAND

ORSANII

PATERNII

PIFDIVAILE

PIFDIPATERNO

PIEVE TORINA

PINTUG!.IA

POSTA

PUST I GNANO

PUPAGGI

ROCCANULFI

SAMBUCHETA

SCHEGGINO

SLHTOPPO

SERRAVAI.LF DI CHIENTI

SPELLIO

STERPARF

S.ANATUL.LA DI NARCI]

S. LAZZARI

S. MAMII. IANU

S. VALENT INO

TFRNF.

TERRIA

VAI LO DI NFRA

UILLAMAGINA

UII LA DEL GUADL

AMATRICE

ANTRUDOCO

APPENN [N1]

AROUATA DEL. TRONTO

ARRLINF

BFLMUNTE IN SABINA

BOL OGENOI A

CAI DAROL.A

CAL UI

CAMFRIND

CAMPELI.I) SUL RLITUNNI

CAMPOTOSTO

CASPERIA

CASTEI RAJMUNDO

CASTEI S.FEIILE

CASTEI LUCCIO

CITTADILATE

COI FIUR I TO

CULIE SOGLIO

COILFSTATTE

CONLERUIANO

CONF IGN I

Ebis:

FIUMINATA

FULJIENO

FRANCAUILLA O'ETE

GAVELLII

GRFICLIO

GUAI DU

LAORO

LONISONF. SAAINO

MUNTEFA'.CO

MUNTERFAIE

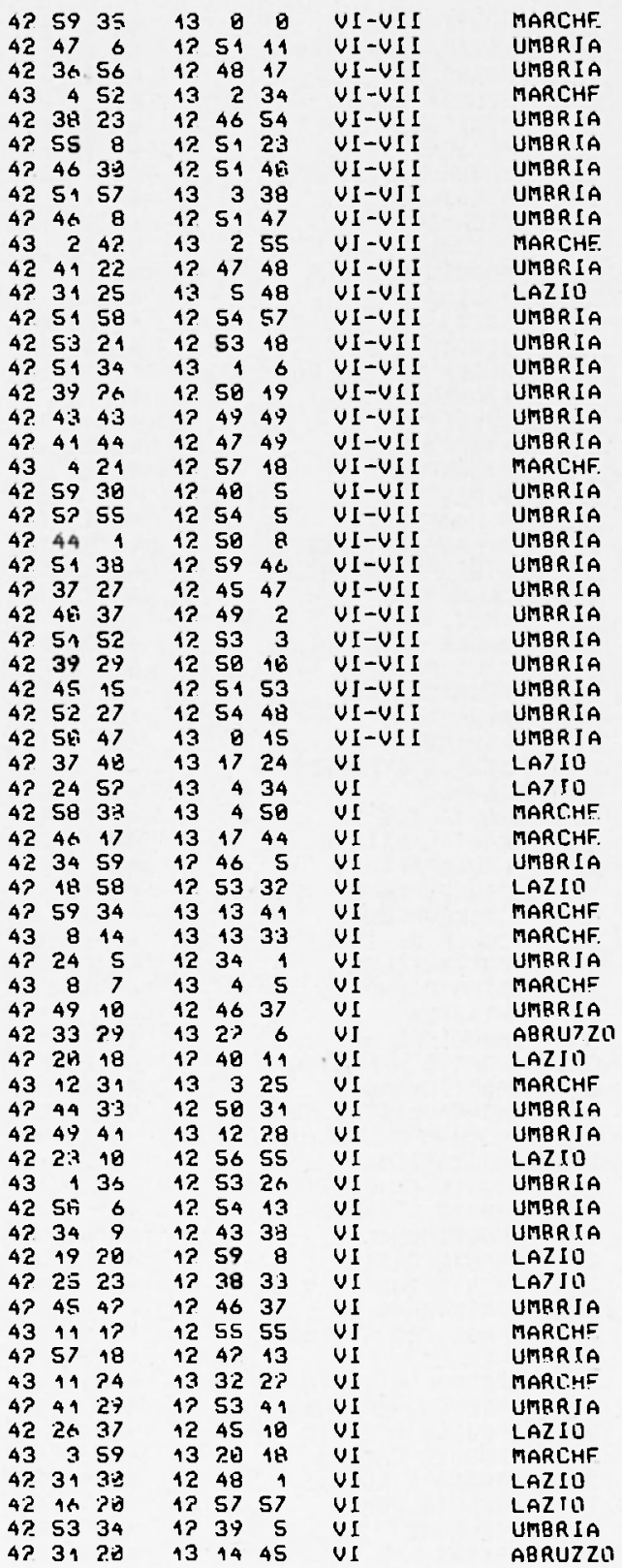




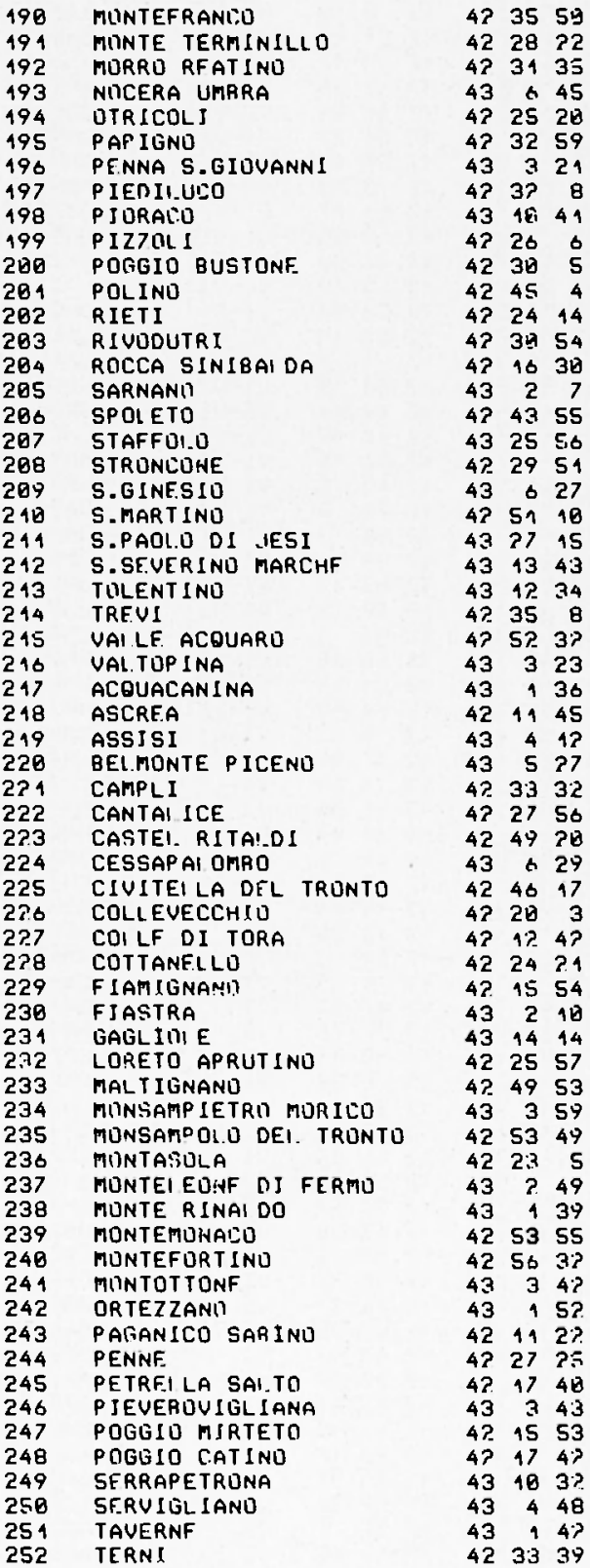

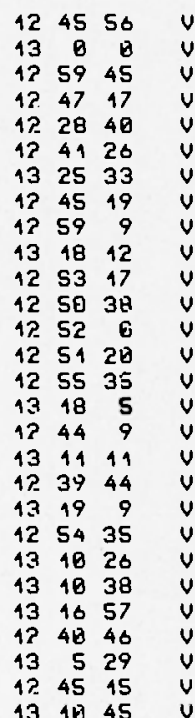

131045

$\begin{array}{lll}12 & 59 & 46 \\ 12 & 36 & 58\end{array}$

$\begin{array}{lll}13 & 32 & 22\end{array}$

$1344 \quad 49$

$1254 \quad 45$

12 4023

$\begin{array}{lll}13 & 45 \quad 29\end{array}$

$1340 \quad 3$

12. 3340

125652

$1241 \quad 8$

13734

13948

$\begin{array}{lll}13 & 4 & 4\end{array}$

$1359 \quad 45$

$\begin{array}{lll}13 & 44 & 43\end{array}$

$13 \quad 33 \quad 26 े$

$\begin{array}{llll}43 & 47 & 37\end{array}$

42. 4 B 58

433444

$1334 \quad 47$

$\begin{array}{lll}13 & 49 \quad 35\end{array}$

$13 \quad 2831$

13356

$43 \quad 36 \quad 34$

175958

135539

1345

1355

12. 449

17. 4429

$\begin{array}{lll}13 & 11 & 19\end{array}$

$13 \quad 2931$

12. 554

17. $38 \quad 51$

UI
UI
UI
UI
UI
UI
UI
UI
UI

UMRRJA

LAZIO

LAPII

UMBR IA

UMBRIA

UMBRIA

MARCHE

UMBR I A

MARCHF

ARRUZZO

LAZ1O

UMRRIA

LAZ IO

LAZII

LAZIO

MARCHF

UMBR JA

MARCHF

UMBR IA

MARCHF

UINER IA

MARCHF.

MARCHF

MARCHF

UMBRIA

UMRRIA

UMBR I A

MARCHF

LAZJO

UMBRJA

MARCHF

ABRUZZO

LAZ 10

UMRR JA

MARCHF

ABRIIZ7.0

LA7 10

LAZIO

LAZ10

LAZ10

MARCHF

MARC.HE

ARRUZZO

FARC.HE

MARI:HE

MARCHE

LAZIO

MARC.HE

MARCHF

MARCHF

MARCHF

MARCHF

MARCHE.

LAZIO

ARRU7ZO

LAZIO

MARCHF

LA7 10

LAZIO

MARCHF

MARCHF

MARCHF

UMBRJA 
NUM.

INT.

REGIONE

USSITA

VAL.LE CASTEI LANA

ACQUUASPARTA

AI ANNII

AI.8A ARR IATICA

AMANDOLA

AMFL IA

APP I GNANO

ASCOI.I PICENI

ATESSA

ATR I

AUETZANI

BASSANI IN TEVERINA

BASTIA UMBRA

BELLANTE

BETTUNA

BEVAGNA

BOMBA

BURRFLI_O

BULCHIANICO

CAMFRANU

CAMPURUTUNDO FIASTRONF.

CANNARA

CAPRARULA

CARROGNANI

CARSOLI

CASTEL COLONNA

CAT I GNANII

CELANO

CERRFTO D'ESI

CINGOLI

CIUITA CASTELL ANA

COLLAZZONE

CUI MURANU

COL ONNFLL.A

COMIINANZTA

CORCH IANI]

CURRIDUNIA

CURUARU

COSSIGNANU

CROGNAL ETO

DERUITA

ESANATOG!.IA

FABRICA OI ROMA

FALERIA E CALCATA

FARA SABINA

FORCF.

FRASSO

FRATTA TUOINA

GENLIA

GIANO DEI.L'UMBRIA

GIUVE

GRAFF I GNANO

GROT TAMMARE

GUAI DO TADINO

GUAL DU CAT TANFO

GUARIDEA

ISULA DEL. GRAN SASSO

$4256 \quad 45$

$13 \quad 838$

$U-U I$

$\begin{array}{lll}42 & 44 & 6 \\ 42 & 41 & 24\end{array}$

$42 \quad 17 \quad 37$

$\begin{array}{lll}42 & 49 & 37\end{array}$

$\begin{array}{lll}42 & 58 & 47\end{array}$

$42 \quad 33 \quad 28$

432149

$4251 \quad 1 ?$

42358

$4234 \quad 4$ H

$42 \quad 153$

$42 \quad 2754$

$43 \quad 353$

424436

43 हो 45

425555

$42 \quad 25$

$4155 \quad 7$

$\begin{array}{lll}42 & 18 & 14\end{array}$

$\begin{array}{llll}43 & 31 & 47\end{array}$

43752

$4 ? 5939$

$41 \quad 1936$

42195 ?

42551

434 A $^{4} 43$

$42 \quad 20 \quad 45$

4 ? 51

$\begin{array}{lll}43 & 19 & 43\end{array}$

432723

$\begin{array}{lll}42 & 17 & 34\end{array}$

$4253 \quad 53$

43948

4? 5? 18

4 ? 5730

$4220 \quad 45$

43 14 52

4? $12 \quad 19$

$42 \quad 58 \quad 59$

42 3ล 41

$42 \quad 58 \quad 54$

$\begin{array}{lll}43 & 15 \quad 4\end{array}$

$42 \quad 202$

$\begin{array}{llll}42 & 13 & 3.3\end{array}$

42. 12. 31

$\begin{array}{lll}42 & 57 \quad 45\end{array}$

$42 \quad 13 \quad 44$

4 ? 5125

$43 \quad 2544$

$\begin{array}{lll}42 & 49 & 57\end{array}$

42. $34 \quad 32$

$42 \quad 34 \quad 23$

$4258 \quad 47$

$43 \quad 93 \quad 48$

4? 5433

$\begin{array}{lll}42 & 37 & 19\end{array}$

42364

$\begin{array}{lll}13 & 29 & 5\end{array}$

$\begin{array}{lll}12 & 32 \quad 47\end{array}$

$1358 \quad 16$

$1355 \quad 49$

132425

$12 \quad 24 \quad 45$

132047

$1334 \quad 41$

$\begin{array}{lll}14 & 26 & 45\end{array}$

135848

$\begin{array}{lll}13 & 25 & 32\end{array}$

124840

12336

$\begin{array}{lll}13 & 48 & 21\end{array}$

$12 \quad 296$

$12 \quad 36 \quad 28$

$14 \quad 2159$

$14 \quad 18 \quad 16$

$14 \quad 16 \quad 53$

$13 \quad 33 \quad 4$

13 is 53

123459

$\begin{array}{lll}12 & 14 & 17\end{array}$

12. 160

1353

$13 \quad 6 \quad 25$

$\begin{array}{lll}13 & 572\end{array}$

$\begin{array}{lll}13 & 32 & 43\end{array}$

12 596

$13 \quad 1258$

172445

$\begin{array}{lll}12 & 26 & 8\end{array}$

$\begin{array}{lll}13 & 21 & 28\end{array}$

135159

132454

$\begin{array}{lll}12 & 2127\end{array}$

133036

$\begin{array}{lll}13 & 16 \quad 2\end{array}$

$\begin{array}{llll}13 & 41 & 15\end{array}$

13 29 24

$12 \quad 25 \quad 1$ हे

12 5652

$\begin{array}{lll}12 & 17 & 43\end{array}$

$12 \quad 26 \quad 35$

$12 \quad 4346$

$\begin{array}{lll}13 & 29 & 27\end{array}$

$\begin{array}{lll}12 & 48 & 17\end{array}$

12. 2157

12506

17. 3446

$\begin{array}{lll}12 & 19 & 27\end{array}$

1? 1? 17

$\begin{array}{lll}13 & 52 \quad 19\end{array}$

17. 477

17 $33 \quad 2 \%$

12. 1753

$13 \quad 3939$

$1325 \quad 8$

LURL PICENO

432344

$\begin{array}{lll}13 & 27 & 8\end{array}$

MACERATA

MALI IANII SAB INA

345 MAGLIANO DI TENNA

MARCHF

ABRUZZI]

UMBRIA

ABRUZZO

ABRU7.70

MARCHF

UMRRIA

MARCHE

MARCHF.

ARRU7 ZO

ARRU77.1

ARRUZZO

LA7.10

UMRRIA

ARRUZZO

UMBRIA

UMBRIA

ARRUZZU

ARRUZ70

ABRUZ7O

MARCHF

MARC.HF

UMARIA

LA7. IO

LAZIU

ABRII7:Z0

MARCHF

ARRU7 ZO

ABRU7ZO

MARC.HF

MARCHE

LAZIO

UMBRSA

MARCHF

ABRUZ7II

MARC.HF.

LAZ IO

MARCHF

LAZ IU

MARCHIF.

ABRU7.7n

UMBR I A

MARCHF

LAZIU

LAZIO

LAZIU

MARCHE

LA7. IU

UMRR IA

MARCHF

UMRRJA

UMBR IA

LAZIO

MARCHE

UMBR S A

UMBRIF

UMBR IA

ARRUZZO

MAREHF

ARRUZZO

MAREHF

LAZIO

MARLHF 
VIII

$\begin{array}{lll}\text { NUM. LOCALITA' LAT. NORD LONG. EST INT. REGIONE } & \end{array}$

MASSA MARTANA

$42 \quad 46 \quad 31 \quad 12 \quad 3138 \quad v$

MATEL JC:A

MIIGLIANO

MUMPEO

MUNTE S.UITO

MONTEI UPONF

MUNTEGIORGIO

MUNTECASTRILI_I

MUNTE S. MARTINO

MUNTEFAI CONF. APPENNINO

MUNTECASSIANO

MUNTEL.PARO

MONTEFANU

MONTECAROTTO

MUNTE URANO

MUNTOPOLI

MUNTORIO AI VUMANU

MURROUA!LI.E

MUSCIANU S. ANGBEI.C

M.CASTEI_LO DI UIBIO

NARNI SCAI.O

NARN I

NFP I

NFRFTO

NFRITO

ORIOI.O ROMANO

ORTE

ORTUCCHIO

OUINDOLI

PASSO CORFSF

PENNA S. ANDRFA

PERUIIIA

PESARO

PETRIGNANu D'ASSISI

PETRITOLI

PETRIOLO

P J ANFLLLA

PJETRACAMFLA

PJGLIO

POGGIO NATIUO

POI LFNZA

PONTE S.GIOUANNS

POPOI I

PORANO

RIPATRANSONF

RIPE

ROCCA DI MFTZO

RONETGLIONF

SAL I SANII

SANIIFMINI

SCANDRIGLIA

SCHEGEIA

SFFRO

SGURGOLA

SMERILIO

SORA

SP INA

STIMIGLIANU

S. BENFDETTO DEI. TRONTO

$43 \quad 152$

$43 \quad 11$

$\begin{array}{llllll}42 & 14 & 51 & 12 & 45 & 9\end{array}$

$\begin{array}{llllll}43 & 36 & 2 & 13 & 16 & 14\end{array}$

$\begin{array}{llllll}43 & 20 & 36 & 13 & 34 & 5\end{array}$

$\begin{array}{llllll}43 & 7 & 49 & 13 & 32 & 14\end{array}$

$42 \quad 3859$

$43 \quad 149$

$\begin{array}{lll}12 & 29 & 11\end{array}$

$\begin{array}{lllllll}42 & 59 & 13 & 13 & 27 & 34\end{array}$

$\begin{array}{llllll}43 & 24 & 36 & 13 & 25 & 13\end{array}$

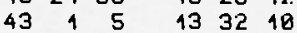

$\begin{array}{llllll}43 & 24 & 41 & 13 & 26 & 17\end{array}$

$\begin{array}{llllll}43 & 31 & 33 & 13 & 3 & 48\end{array}$

$\begin{array}{llllll}43 & 12 & 8 & 13 & 40 & 21\end{array}$

$\begin{array}{llllll}42 & 14 & 48 & 12 & 41 & 28\end{array}$

$\begin{array}{llllll}42 & 34 & 56 & 13 & 37 & 43\end{array}$

$\begin{array}{llllll}43 & 18 & 58 & 13 & 34 & 48\end{array}$

4? 4454

$4256 \quad 24$

423155

$4231 \quad 1$

4 ? 14 2\%

$\begin{array}{lll}42 & 49 & 7\end{array}$

$42 \quad 32 \quad 48$

4 ? 930

42. $27 \quad 34$

$\begin{array}{lll}41 & 57 \quad 13\end{array}$

4288

47916

$42 \quad 35 \quad 34$

$\begin{array}{lll}43 & 6 & 22\end{array}$

$435 \uparrow 16$

43 o 9

$\begin{array}{lll}43 & 4 & 0\end{array}$

$\begin{array}{llll}43 & 13 & 14\end{array}$

$42 \quad 2352$

$42 \quad 3123$

41494 ?

$421 ? 48$

$\begin{array}{llll}43 & 16 & 1\end{array}$

$43 \quad 5 \quad 19$

42. $18 \quad 14$

$4241 \quad 10$

42. 5950

$43 \quad 46 \quad 16$

$\begin{array}{llll}42 & 17 & 19\end{array}$

$\begin{array}{llll}42 & 17 & 27\end{array}$

$\begin{array}{lll}13 & 53 & 48\end{array}$

$\begin{array}{lll}12 & 24 & 8\end{array}$

12313

$1231 \quad 16$

12 2E 4?

$\begin{array}{lll}13 & 49 & 7\end{array}$

$1328 \quad 43$

12821

$12 \quad 235$

133838

133856

$1238 \quad 49$

$1346 \quad 24$

$17 \quad 23 \quad 11$

$1254 \quad 18$

$\begin{array}{lll}12 & 32 & 8\end{array}$

$13 \quad 3927$

132750

1430

$13 \quad 33 \quad 14$

13841

12. $47 \quad 34$

132053

$\begin{array}{lll}12 & 26 & 12\end{array}$

$\begin{array}{lll}13 & 49 & 58\end{array}$

1268

$13 \quad 45 \quad 43$

$\begin{array}{lll}13 & 6 & 24\end{array}$

$\begin{array}{llll}13 & 31 & 14\end{array}$

$\begin{array}{lll}17 & 13 & 3\end{array}$

$\begin{array}{llll}4 & 17 & 44 & 40\end{array}$

$\begin{array}{llllll}47 & 36 & 46 & 12 & 32 & 47\end{array}$

$\begin{array}{llllll}47 & 9 & 53 & 12 & 58 & 32\end{array}$

$\begin{array}{llllll}43 & 24 & 8 & 17 & 48 & 3\end{array}$

43 8 44 12 5656

$\begin{array}{llllll}41 & 42 & 13 & 13 & 8 & 56\end{array}$

$43 \quad 13 \quad 13264$ ?

$\begin{array}{llllll}41 & 43 & 6 & 13 & 36 & 47\end{array}$

$\begin{array}{llllll}42 & 59 & 7 & 12 & 16 & 49\end{array}$

$\begin{array}{llllll}42 & 17 & 50 & 17 & 33 & 55\end{array}$

$\begin{array}{llllll}42 & 56 & 1 & 13 & 53 & 34\end{array}$

$\begin{array}{llllll}42 & 49 & 39 & 13 & 4 & 55\end{array}$

S.EIPIDIO A MARF.

13410

S. MARIA DEGLI ANGEII

S. UITTURIA MATENANU

43338

$17 \quad 34 \quad 45$

$13 \quad 2945$

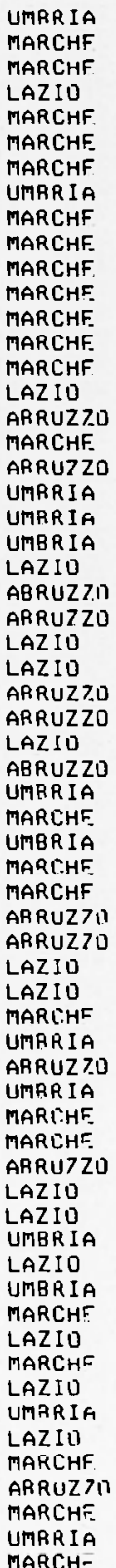


NUM. LOCALITA'

LAT. NORD LONG. EST

INT.

REGIONE

\begin{tabular}{|c|c|}
\hline $\begin{array}{l}379 \\
380 \\
381 \\
382 \\
383 \\
384 \\
385 \\
386\end{array}$ & $\begin{array}{l}\text { S.UITU RUTANO } \\
\text { TERAMU } \\
\text { TODI } \\
\text { TURGIANU } \\
\text { TURTORETU } \\
\text { URB ISAGLIA } \\
\text { UAL.FARBRICA } \\
\text { UAI_LF.RANU }\end{array}$ \\
\hline & $\begin{array}{l}\text { VASANELLL } \\
\text { VIGNANELLLO }\end{array}$ \\
\hline $\begin{array}{l}389 \\
390\end{array}$ & $\begin{array}{l}\text { UILLI.A S. LUCIA D.ARRUZZI I } \\
\text { ACOUASANTA TERMF }\end{array}$ \\
\hline & $\begin{array}{l}\text { AL.ATRI } \\
\text { AI.TINU }\end{array}$ \\
\hline & $\begin{array}{l}\text { ANAGNI } \\
\text { ARP INU }\end{array}$ \\
\hline & $\begin{array}{l}\text { CANFP INA } \\
\text { CASACANDITEI.LA }\end{array}$ \\
\hline $\begin{array}{l}397 \\
398\end{array}$ & $\begin{array}{l}\text { CASAI-INCONTRADA } \\
\text { CASOLI }\end{array}$ \\
\hline 399 & CASTEI LI \\
\hline 480 & CASTEI S.EL IA \\
\hline 401 & CASTEI DEL. MUNTE \\
\hline 402 & CASTEI PLANIO \\
\hline 403 & CASTELINUUUU UUMANU \\
\hline 404 & CEPRANU \\
\hline 405 & CHIETI (SCAI-U) \\
\hline 406 & CIUITAQUANA \\
\hline 407 & CIUITELLA CASANUVA \\
\hline 408 & COLIESCIPOLI \\
\hline 489 & CONTIGLIANU \\
\hline 410 & CORCIANU \\
\hline 419 & CORROPOLI \\
\hline 412 & CUPRAMUNTANA \\
\hline 413 & FILOTTRANU \\
\hline 414 & FUSSATO DI UICO \\
\hline 415 & FROSINUNF \\
\hline 416 & GRECCIO \\
\hline 417 & GUBB IU \\
\hline 418 & MFREO \\
\hline 419 & MUNTAL TO MARCHE \\
\hline 420 & MUNTE GIBERTO \\
\hline 421 & MUNTEMARCIANU \\
\hline 422 & MONIE UIDUN COMBATTE \\
\hline 423 & MUNTE S.PIETRANIAEL. I \\
\hline 424 & MUNTEROSI \\
\hline 425 & MUNTEPRANDUNF \\
\hline 426 & MUNTERADU \\
\hline 427 & MURRO D.AI.BA \\
\hline 428 & NUTARFSLO \\
\hline 429 & URSUGNA \\
\hline 430 & PALMIANO \\
\hline & PUGGIO S.MARCELI.O \\
\hline 432 & PON7ANII DI FERMO \\
\hline & PURTO S.GIURGIO \\
\hline 43 & RAPAGNANU \\
\hline & RUSGAA \\
\hline & SIGILI.0 \\
\hline & SURIANO NFL CIMINU \\
\hline & SUTRI \\
\hline & S.MARCEI.LO \\
\hline & TAGL IACOZ70 \\
\hline & VENAKOITA \\
\hline
\end{tabular}

$\begin{array}{rrr}41 & 52 & 53 \\ 42 & 39 & 31 \\ 42 & 46 & 53 \\ 43 & 1 & 29 \\ 42 & 48 & 17 \\ 43 & 11 & 45 \\ 43 & 9 & 29 \\ 41 & 53 & 17 \\ 42 & 24 & 56 \\ 42 & 23 & 9 \\ 42 & 19 & 57 \\ 42 & 46 & 9 \\ 41 & 43 & 32 \\ 42 & 6 & 6 \\ 41 & 44 & 31 \\ 41 & 38 & 59 \\ 42 & 27 & 59 \\ 42 & 14 & 54 \\ 42 & 17 & 27 \\ 42 & 37 & 9 \\ 42 & 17 & 44 \\ 42 & 14 & 57 \\ 42 & 21 & 49 \\ 43 & 29 & 34 \\ 47 & 37 & 49 \\ 41 & 32 & 49 \\ 42 & 21 & 23 \\ 42 & 19 & 28 \\ 42 & 21 & 56 \\ 42 & 32 & 11 \\ 42 & 24 & 39 \\ 43 & 7 & 49 \\ 42 & 49 & 41 \\ 43 & 26 & 42 \\ 43 & 26 & 4 \\ 43 & 17 & 42 \\ 41 & 33 & 8 \\ 42 & 26 & 37 \\ 43 & 21 & 5 \\ 43 & 28 & 17 \\ 42 & 59 & 16 \\ 43 & 5 & 26 \\ 43 & 38 & 19 \\ 43 & 2 & 59 \\ 43 & 11 & 29 \\ 47 & 11 & 44 \\ 42 & 55 & 9 \\ 43 & 41 & 49 \\ 43 & 36 & 4 \\ 42 & 39 & 26 \\ 47 & 13 & 6 \\ 42 & 53 & 55 \\ 43 & 36 & 40 \\ 43 & 6 & 6 \\ 43 & 16 & 49 \\ 43 & 7 & 33 \\ 43 & 28 & 56 \\ 43 & 19 & 54 \\ 42 & 25 & 5 \\ 47 & 14 & 36 \\ 43 & 34 & 31 \\ 47 & 4 & 4 \\ 42 & 52 & 52 \\ 4 & & \end{array}$

17 $58 \quad 46$

$\begin{array}{lll}13 & 42 \quad 14\end{array}$

$\begin{array}{lll}12 & 24 & 24\end{array}$

17207

13 Sn 49

$\begin{array}{lll}13 & 27 & 37\end{array}$

$1236 \quad 8$

17 $58 \quad 24$

17 2044

$12 \quad 16 \quad 44$

$\begin{array}{lll}13 & 46 \quad 37\end{array}$

132436

132038

$\begin{array}{lll}14 & 19 & 59\end{array}$

$1392 \%$

$\begin{array}{lll}13 & 36 & 37\end{array}$

$1214 \quad$

$\begin{array}{lll}14 & 1159\end{array}$

1484

$1358 \quad 48$

$\begin{array}{lll}14 & 25 & 47\end{array}$

$12 \quad 2210$

$1343 \quad 35$

13456

13513

13310

1482 ;

135357

$13 \quad 53 \quad 19$

12. $37 \quad 11$

$1746 \quad 8$

$1217 \quad 13$

134957

13 \% 58

13213

124543

132025

124518

1.2 3438

132 i?

$13 \quad 36 \quad 30$

133750

$\begin{array}{lll}13 \quad 18 & 37\end{array}$

$13 \quad 3752$

$13 \quad 34 \quad 41$

$\begin{array}{lll}12 & 18 & 31\end{array}$

13506

13531

$\begin{array}{lll}13 & 97 & 49\end{array}$

$13 \quad 53 \quad 39$

$14 \quad 1657$

1327 उधे

$\begin{array}{lll}13 & 4 & 35\end{array}$

$13 \quad 39 \quad 31$

$\begin{array}{lll}13 & 47 \quad 37\end{array}$

133536

1348

$12 \quad 44 \quad 27$

$12 \quad 14 \quad 6$

$\begin{array}{lll}12 & 13 & 27\end{array}$

$134 ? 24$

13 15 3

132934 $u$
$u$
$u$
$v$
$v$
$v$
$u$
$u$
$u$
$u$
$u$
$I v-v$

IV-V

IV-V

IV $-V$

IV $-\mathrm{V}$

IV-V

IV-V

IV-V

IV-V

I $v-v$

I $V-V$

I $v-v$

IV -4

IV-V

IV-V

IV $-V$

IV $-V$

IV $-V$

IV-V

IV-V

IV-V

I $V-V$

I $V-V$

IV $-V$

IV $-\mathrm{V}$

IV-V

IV $-\mathrm{V}$

IV $-\mathrm{V}$

IV-V

IV $-V$

IV $-V$

IV $-V$

IV $-\mathrm{V}$

I $v-v$

IV $v$

IV $-U$

I $V-V$

IV $-V$

IV $-U$

I $V-V$

IV $-V$

IV-V

IV-V

IV-U

IV $-V$

IV $-V$

IV $-U$

IU $U$

IV $-V$

IV $-V$

IV $-V$

I $U-1)$
LAZ10

ARRUZ7O

UMBR IA

UMRR IA

ARRUZ7O

MAREHF

UMRRIA

LA7 IO

LA7 IO

LAZ 10

ARRUZ7O

MARCHF

LAZIO

ARRUZZO

LA7 10

LA7 I0

LAZIO

ARRUZZO

ARRUZ7.0

ARRUZ7 7

ARRUZ7O

LAZ IO

ARRUZ7.0

MAREHE

ARRUZ7.

LAZIO

ARRUZ7.0

ARRUZ70

ARRUZZO

UTRRIA

LAZIO

UTBRIA

ARRUZ7N

MAREHF.

MARCHF

UMBAIA

LAZIU

LAZIO

UMBRIA

MARËHF

MARCHF.

TARCHF

MARCHF

MARCHE

MARLHF

LAZIO

MAREHF

MARËHF

MARCHF.

ARRUZZU

ARRUZ 71

MARLHE

MARLHE

MARLHF.

MARLHE

MARLHF

MARCHF

UTBR I A

LAZIO

LAZIO

MARCH

ARRUZZU

MAYCHF 
NUM. 'OOCALITA

LAT. NORD

LONG. EST

INT.

REGIONE

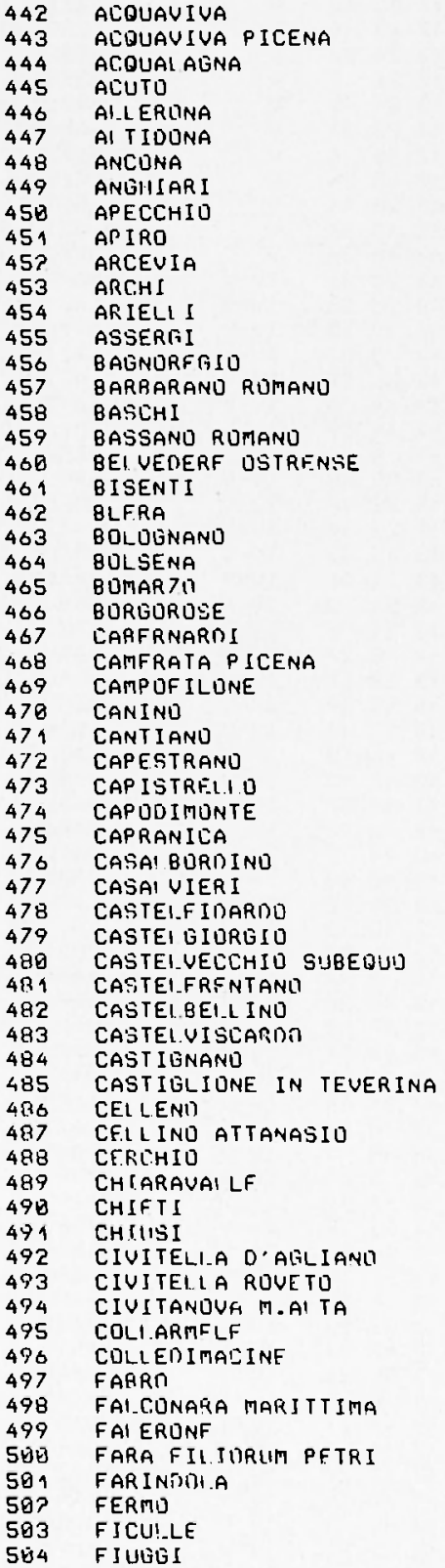

$\begin{array}{rrr}43 & 23 & 2 \\ 47 & 50 & 38 \\ 43 & 37 & 10 \\ 41 & 47 & 23 \\ 42 & 48 & 41 \\ 43 & 6 & 26 \\ 43 & 36 & 11 \\ 43 & 32 & 25 \\ 43 & 33 & 32\end{array}$

433339

$\begin{array}{lll}43 & 23 & 27\end{array}$

432755

$42 \quad 5 \quad 19$

$42154 ?$

47 $24 \quad 49$

47 $37 \quad 34$

47 152

47467

42136

43 34 46

423148

$42 \quad 1621$

$42 \quad 13 \quad 1$

$\begin{array}{llll}42 & 38 & 37\end{array}$

$42 \quad 29 \quad 15$

$42 \quad 1136$

$43 \quad 3024$

$43 \quad 34 \quad 37$

43442

$42 \quad 27 \quad 55$

432 2 27

$42 \quad 15 \quad 5 \%$

415757

$42 \quad 32 \quad 59$

$\begin{array}{lll}42 & 15 \quad 23\end{array}$

$42 \quad 859$

413751

$43 \quad 27 \quad 48$

47. 4729

47. 746

421149

4327 1ำ

$\begin{array}{lll}47 & 45 \quad 17\end{array}$

47 $56 \quad 14$

42. $38 \quad 45$

$42 \quad 3727$

47358

$47 \quad 349$

43 35 4

43214

43 5 55

47 $36 \quad 18$

41 5月 49

$43 \quad 18 \quad 55$

47333

42613

47. 5147

$43 \quad 37 \quad 35$

43624

471455

$42 \quad 20 \quad 25$

$\begin{array}{lll}43 & 9 & 37\end{array}$

42516

1110

$1348 \quad 48$

1? 40 21

$13 \quad 1032$

115825

$1347 \quad 46$

13 3日 26

17. 310

$\begin{array}{lll}12 & 25 & 11\end{array}$

13754

1? $50 \quad 27$

142753

$14 \quad 18 \quad 26$

$\begin{array}{lll}13 & 36 & 18\end{array}$

12. 542

1?. 357

121258

1? 1137

13 但

$\begin{array}{lll}13 & 48 & 7\end{array}$

$17 \quad 139$

$1357 \quad 37$

$1159 \quad 13$

17 152

$1314 \quad 3$

1?. 5159

13219

134851

114459

$1237 \quad 41$

$1346 \quad B$

$\begin{array}{lll}13 & 23 & 27\end{array}$

$1154 \quad 44$

1? 14 36

14352

13 4? 43

13336

115844

1343517

$\begin{array}{lll}14 & 21 & 17\end{array}$

13847

1206

$13 \quad 37 \quad 18$

$12 \quad 17 \quad 13$

1282

135134

13364

$\begin{array}{lll}13 & 19 & 33\end{array}$

14 1ह 6

$115050^{\circ}$

1? 1110

13 2528

134051

1337 32

$14 \quad 17 \quad 3$

$17 \quad 947$

132350

$\begin{array}{lll}13 & 28 & 19\end{array}$

14119

134924

134256

17 357

$1313 \quad 27$

IV
$I V$
$I V$
$I V$
$I V$
$I V$
$I V$
$I V$
$I V$
$I V$
$I V$
$I V$
$I V$
$I V$
$I V$
$I V$
$I V$
$I V$
$I V$
$I V$
$I V$
$I V$
$I V$
$I V$
$I V$
$I V$
$I V$
$I V$
$I V$
$I V$
$I V$
$I V$
$I V$
$I V$
$I V$

TUSCANA

MARCHF

MARCHF

LAZIO

UMBRIA

MAREHF.

TARCHF.

TOSIANA

TAREHF

TAREHF

MARCHF

ARRUZ71

ARRUZ70

ARRUZ7.0

LAZ IO

LAZ IO

UMBRIA

LA7 IO

MAREHF.

ARRUZZ i

LAZIU

ARRUZZO

LA7 IO

LAZIO

LAT IO

MARCHF

MARLHF

TAREHF

LAZ IO

MAREHF

ARRUZZO

ARRUZ7 0

LA? IO

LAZIO

ARRUZ70

LAZ I U

MARC.HF

UTGR IA

ARRUZZO

ARRUZZ

MARCHF

UTARIA

MARCHF

LAZIO

LAZIU

ARRU7Z1

ARRU7 70

TAREHE

AFRU7ZO

TOSLANA

LAZIO

ABRRUZ717

TARCHF

ARRU7ZO

ARRUZ717

UMRRIA

MAREHE

TAREHF

ARRU721

ASRU770

TARCHF

UMER I A

LA7 II 
NUM.

505 FUNTECCHIO

506 FOSSACFSIA

507 FRANCAUILI.A MARE

FRASCAT I

GAL.LESE

510 GESSUPAL.FNA

511 GIOIA DEI MARSI

512 GIUI.IANOUA

513 GORIANO SICOLI

514 GROTTA7ZOLINA

515 GROTTE DI LASTRO

516 GUAKDIFGRFLF.

517 ISFRNIA

518 ISOLA DEL. LIRI

519 JESI

520 LAMA DEI PEIIIGNI

521 LANL:IANIT

522 LAPEDONA

523 LORETO

574 LUCO DEI MARSI

5.25 LUGNANU IN TEUERINA

526 MAIOLATI SPONTINI

MANUPPEI. LO

MARINO

MARSCIANO

MARTA

MAR I INSILURO

MASSA FERMANA

MIGI_IANICO

MIINSANU

MUNTAPPONF

MIINTEIIRANARO

MONTEFIORF DEI_L'ASO

MUNTERAGRTONE

MIONTE UIDUAN CORRADO

MUNTEI EQINE D'ORUIETO

MONTE PQR7IU C.ATONE

MUNTEF IASLONE

MUNTEC.CHIO

MITNTEROOSART

MIINTERUAERTO

MIINTERCHI

MUNTENERODOM!]

MLINTERIIRB I ANO

MUNTE S.gIUSTO

MURESCI

NAVEI LI I

NIIMANA

OFFAGNA

ORTONA

ORTUNA DES MARSI

ORUIFTO

OSIMU

OSTRA

PAGI_IETA

PAI ENA

PAI. IANLI

PARRANI

PEDASO

PENNFIDITU

PESLARA

$42 \quad 13 \quad 49$

$13 \quad 36 \quad 19$

IV

142858

$\begin{array}{lll}41 & 48 & 27\end{array}$

42 2ं 2 म

124051

$\begin{array}{lll}12 & 40 & 51 \\ 12 & 24 & 18\end{array}$

$\begin{array}{llllll}3 & 3 & 18 & 14 & 16 & 24\end{array}$

$\begin{array}{llllll}41 & 57 & 11 & 43 & 41 & 33\end{array}$

$\begin{array}{llllll}42 & 45 & 5 & 13 & 57 & 28\end{array}$

$42 \quad 4 \quad 47$

$1346 \quad 29$

$\begin{array}{llllll}43 & 6 & 4 & 13 & 36 & 11\end{array}$

42 4र: 27

$\begin{array}{lll}42 & 1124\end{array}$

413537

41 AE 49

4331213

$42 \quad 2 \quad 29$

$\begin{array}{lll}42 & 13 & 49\end{array}$

$\begin{array}{lll}43 & 63\end{array}$

$43 \quad 26 \quad 2 ;$

$4157 \quad 32$

$4 ?$ 3? 24

43 2. 34

42. 15 PS

$\begin{array}{llll}41 & 46 & 11\end{array}$

$\begin{array}{llll}42 & 54 & 34\end{array}$

4? 325

42534

43855

$42 \quad 213$

$\begin{array}{lll}43 & 3: 3 & 46\end{array}$

$438 \quad 14$

$\begin{array}{lll}43 & 13 & 57\end{array}$

4334

$\begin{array}{lll}42 & 55 & 13\end{array}$

43716

42. 552

414855

$42 \quad 32 \quad 14$

$\begin{array}{lll}42 & 39 & 4: 3\end{array}$

$\begin{array}{lll}43 & 18 & 53\end{array}$

432859

43275

415834

4356

43,149

4356

$47 \quad 14 \quad 16$

$43 \quad 39 \quad 43$

$43 \quad 3137$

47 $21 \quad 16$

41595 이

42436

$\begin{array}{lll}43 & 29 \quad 7\end{array}$

$43 \quad 36 \quad 44$

42954

41593

$4148 \quad 19$

425146

43549

42 i 16

472749

$1452 \quad 21$

$\begin{array}{lll}14 & 13 & 19\end{array}$

$14 \quad 1350$

$1334 \quad 4$ ?

$13 \quad 14 \quad 41$

$\begin{array}{lll}14 & 11 & 16\end{array}$

$\begin{array}{ll}14 & 2324\end{array}$

$\begin{array}{ll}1346 & 20\end{array}$

1336 3s

$43 \quad 28 \quad 14$

12. 1952

13713

14335

$12 \quad 39 \quad 39$

$\begin{array}{lll}12 & 20 & 47\end{array}$

115531

$\begin{array}{lll}13 & 54 & 49\end{array}$

$13 \quad 28 \quad 32$

$\begin{array}{lll}14 & 17 & 34\end{array}$

$\begin{array}{lll}13 & 15\end{array}$

$13 \quad 2814$

$\begin{array}{lll}13 & 37 & 58\end{array}$

$\begin{array}{lll}13 & 45 \quad 3\end{array}$

$12 \quad 535$

$1329 \quad 8$

1234

12 4? 54

$\begin{array}{lll}12 & 149\end{array}$

$\begin{array}{lll}12 & 179\end{array}$

$\begin{array}{lll}13 & 38 & 10\end{array}$

$\begin{array}{lll}13 & 8 & 48\end{array}$

17. 644

$\begin{array}{lll}14 & 15 & 7\end{array}$

$\begin{array}{ll}13 \quad 42 & 59\end{array}$

$13 \quad 35 \quad 42$

134355

$\begin{array}{lll}43 & 43 & 46\end{array}$

$\begin{array}{llll}1.7 & 37 & 17\end{array}$

$1326 \quad 39$

$\begin{array}{lll}14 & 24 & 13\end{array}$

$\begin{array}{lll}13 & 43 & 41\end{array}$

$\begin{array}{lll}12 & 6 & 47\end{array}$

$13 \quad 2857$

13928

$\begin{array}{lll}14 & 29 & 54\end{array}$

$\begin{array}{lll}14 & 8 & 17\end{array}$

$\begin{array}{lll}13 & 3 & 23\end{array}$

12620

135029

$\begin{array}{lll}14 & 19 & 32\end{array}$

$\begin{array}{lll}14 & 12 & 50\end{array}$

$\begin{array}{llllll}42 & 1 & 39 & 13 & 39 & 26\end{array}$

13 8 5\%

ABRUZ70

ABRU770

ABRUZZO

LAZIO

LAZIO

ABRU770

ABRUZZO

ARRUZZ.I

ARRU7ZO

MARCHF

LAT. IO

ARRUZZO

ARRUZ70

LAZIO

MARCHF

ARRUZZO

ARRUZ 70

MARCHE

MARCHF

AARU7.7.0

UMBRIA

MARCHE

ABRUZZO

LA7 IO

UMBRIA

LAZIO

ARRUZ70

MARCHF

ARRUZZO

MARCHE

MARCHF

MARCHF

MARCHE

UTRRIA

MARCHE

UMRR JA

LAZIO

LAZIO

UTBRIA

MARCHE

MARCHF

TOSLANA

ARRUZ ZO

MARC.HF

MARCHF

MARCHF

AFRUZZO

MARC.HF

MARCHF

ARRU7Zn

ARRU7 70

UIMBRIA

MARCHE

MARCHE.

ARRUT ZII

ARRUZZU

LAZIO

UMBRIA

MARCHE

AARUZZ1

ARRUZZO

ARRU7 Z1)

LAZ IO 
568 PINFTO

569 POGGIO S.UICINO

570

POGGIO FIORITO

PORTO S.EIPIOIO

572 PORTO RECANATI

573 POTENTA PICENA

RAP INI

57S RIPE S.GINESIO

576 ROSE.TO DEGI.I ARRUZZI

577 SCURCOLA MARSICANA

578 SELINARO

S79 SERRA DE'CONTI

580 SERRA 5 . QUIRICO

581 SILUI MARJNA

582 SIROI 0

583 SULMUNA

584 S.ANGFLO IN LIZ7IULA

SBS S.ANGELO IN POHTANO

586 S.BENFDETTO DEI MARSI

587 S.EUSANJU DEL SANITRO

588 S.LORENZO NUUUO

589 S.MARTINO A! CIMINO

598 S.MARIA NUUVA

594 S.NICOLO' A TOROINO

592 S.VENANZO

593 TARANTA PELIGNA

594 TORINO OI SANTIRO

595 TORRE S.PATRIZIO

596 TORRICEI.LA PELIIINA

597 TOSSICIA

598 TRASALCO

599 TUSCANIA

600 URBANIA

6B1 URBINO

602 VATONF

683 VAL.ENTANIO

684 VEIANO

GQS VETRAL LA

606 UILIAMALINA

607 UILLA S.MARIA

608 UITERRI

689 VITURCHIANO

610 ALIJGI.IANIT

614 AQUIINO

612 ATINA

613 CIUITELLA M.RAIMUNOO

614 COLROROOLO

615 CORINALDO

616 FARA S.MARTINO

617 MURINO

618 PIANSANO

619 PQLUERIGI

620 PROCFNO

621 ROMA

622 SANSTPOI.ERO

623 SCERNI

624 SGURGOLA

625 SPINAI.F.TOCROMA)

626 STRANGULALAILI

627 S.VITO CHIFTINO

628 TOI.L.O

629 TURRITA OI SIENA

630 UEROLI

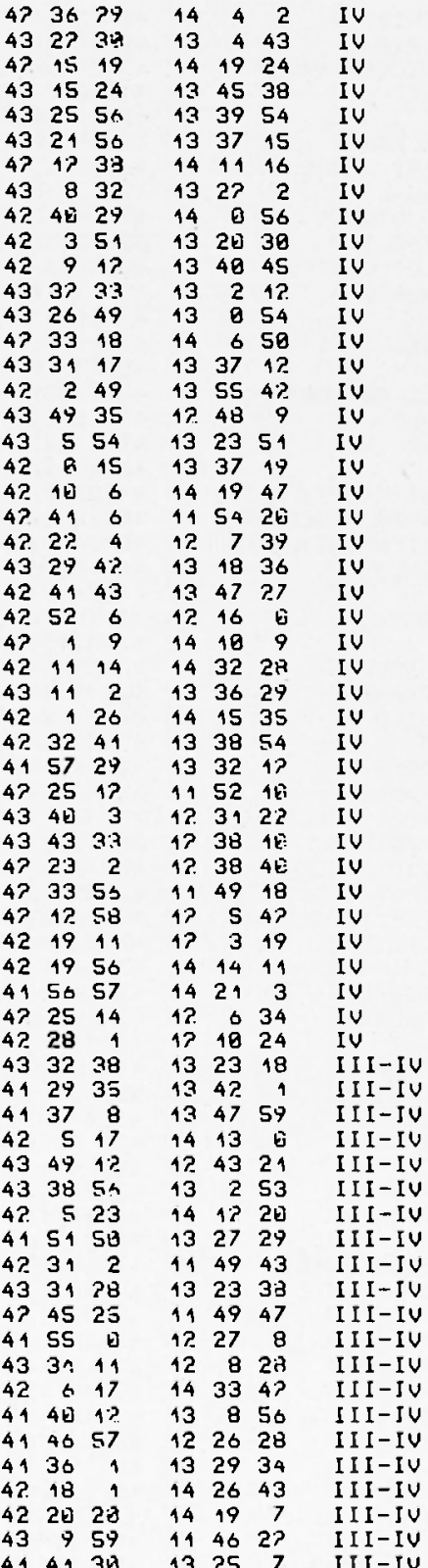

MARCHE

ARRUZ7I

MARC.HE.

MARCHF

MARCHF

ARRUZ70

MARCHF

ARRUZ71]

ARRUIZZO

ARRUZ70

MARCHF.

MARCHF

ARRU7ZO

MARCHF

ARRU7.71

MARCHF.

MARCHF

ARRUZZO

ARRUZ70

LAZIO

LAZ IO

MARCHF

ARRUZZO

UIRRIA

ARRUZZU

ARRUZ7.0

MARCHF

ARRUZZO

ARRUZZO

ARRUZZU

LAZIO

MARCHE

MARCHF

LAZIO

LATIO

LA7 IO

LAZ IO

ABRUZZ.L?

ARRUZZO

LAZ IO

LAZ IU

MARCHF

LAZ IU

LAZIO

ARRUZZO

MARCHF.

MARCHF

ARRUZZO

ARRUZZ1)

LAZIO

MARCHF

LAZIO

LA7IO

TOSCANA

ARRUZT

LA7 IU

LAZ IO

LA7.IO

ARRUZ717

ARRUZZU

TOSCANA

LAZIO 
NUM.

LOCALITA'

LAT. NORD LONG. EST

INT.

REGIONE

631

632

633

634

635

636

637

638

639

640

641

642

643

644

645

646

647

648

649

650

651

652

653

654

655

656

657

658

659

660

661

662

663

664

665

666

667

668
ACOUAPENDFNTE

ACQUAUIVA DI MONTEPUL.

ARCE.

BAL.SORANO

BARAARA

BETTOLI.F

BOUILLE ERNICA

CASSINO

CASTEI LONE DI SUASA

CFTONA

FERFNTINO

LFTTOPAI_FNA

MAR7OCCA

MFRCATEI LO SUL MF.TAURO

MUNTE RUMANO

MONTEMURI_O

MONTEPULCIANO

MORO!_O

ONANO

OSTRA UE.TERE

PALOMRART

PESCOCOSTAN7O

PICO

P IOBB ICO

PONTECORUO

ROCCASECCA

ROCCARASI]

ROCCASCAI ELNA

ROSCIANO

SENIGAL LIA

S.ANGFLO IN VADO

5. CASCIANO gaGn

S.GIOVANNI INLARICO

S. UINCENZO VAL ROVETO

TAROUINIA

CANTAGAI_I 0

PORTO S.STEFANO

S.cascianu val. di pesfi
42443 ?

43655

41356

444829

$43 \quad 34 \quad 45$

$43 \quad 12 \quad 2 \pi$

$4138 \quad 31$

$4129 \quad 18$

$43 \quad 36 \quad 30$

$42 \quad 57 \quad 47$

$\begin{array}{llll}41 & 4127\end{array}$

4206

$4340 \quad 9$

$43 \quad 38 \quad 49$

42 is 1

$43 \quad 27 \quad 27$

43549

$\begin{array}{lll}41 & 38 & 14\end{array}$

$47 \quad 4132$

$4336 \quad 17$

42. 73

4153.20

$4127 \quad 2$

$43 \quad 3549$

$\begin{array}{llll}41 & 27 & 27\end{array}$

$\begin{array}{lll}41 & 33 & 7\end{array}$

415 5 5

$42 \quad 342$

$\begin{array}{lll}42 & 19 & 14\end{array}$

$434 ? 50$

$43 \quad 3751$

425216

$\begin{array}{lll}41 & 39 & 12\end{array}$

414957

$4 ?$ 15 7

$43 \quad 4148$

$4 ? \quad 26 \quad 15$

$\begin{array}{lll}43 \quad 39 & 24\end{array}$

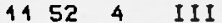

145447 III

133434 III

$\begin{array}{lllll}13 & 33 & 37 & \text { III }\end{array}$

13136 III

$\begin{array}{llll}14 & 48 & 18 & \text { III }\end{array}$

$13 \quad 282 \%$ III

134949 III

12 5838 III

14546 III

$\begin{array}{llll}13 & 15 & 15 & \text { III }\end{array}$

14932 III

$\begin{array}{llll}13 & 48 & 47 & \text { III }\end{array}$

$42 \quad 2943$

145357 III

14732 III

$\begin{array}{lll}14 & 47 & 6\end{array}$

131950 III

144858 III

$\begin{array}{lll}13 & 3 & 32\end{array}$

$\begin{array}{llll}14 & 13 & 51 & \end{array}$

$\begin{array}{lll}14 & 3 & 54\end{array}$

$1333 \quad 35$ III

433641 III

$1340 \quad 5$ III

$4349 \quad 4$ III

14445 III

$\begin{array}{lllll}14 & 18 & 27\end{array}$

14238 III

$\begin{array}{llll}13 & 13 & 23 & \text { III }\end{array}$

$\begin{array}{llll}12 & 24 & 41\end{array}$

$\begin{array}{llll}1152 & 33 & \text { III }\end{array}$

$\begin{array}{llll}13 & 33 & 27 & \text { III }\end{array}$

133128 III

144525 III

$\begin{array}{llll}11 & 13 & 11 & \text { NFG }\end{array}$

$\begin{array}{llll}14 & 7 & 6 & \mathrm{NFG}\end{array}$

$\begin{array}{lll}11 & 146 & 6\end{array}$
LAZIO

TOSCANA

LAZIO

ABRUZZO

MARCHF

TOSCANA

LAZIO

LAZIO

MARCHE.

TOSCANA

LAZ IO

ARRUZZO

MARCHE

MARCHF

LAZIO

TOSCANA

TOSCANA

LAZIO

LAZIO

ARRU7ZO

ARRUZZO

LAZIO

MARCHF

LAZIO

LAZIO

ARRUZZO

ARRUZZO

ARRUZZO

MARCHF

MARCHF.

TOSLANA

LAZIO

ARRUZ70

LAZIO

TOSCANA

TOSCANA

TOSCANA 
TABELLA 3

TIPI DI EDIFICI IN PERCENTUALE;

TIPI DI DANNO IN PERCENTUALE

(secondo la scala MSK) 



\begin{tabular}{|c|c|c|c|c|c|c|c|c|c|}
\hline ASCIA E FRAZIONI & 2 & & 3 & & & & 4 & & \\
\hline & & $A(x)$ & $8(x)$ & $c(x)$ & $1(x)$ & $2(x)$ & $3(x)$ & $4(x)$ & $5[x]$ \\
\hline TRI & 31 & 95 & 5 & -- & 5 & 5 & 25 & 65 & --- \\
\hline AUENDITA & 80 & 20 & 70 & 10 & 30 & --- & 50 & 20 & --- \\
\hline UNA & 18 & 70 & 25 & 5 & 10 & 25 & ss & 10 & --- \\
\hline CAPANNE DI C.GIACONE. & 3 & --- & $\ldots$ & 100 & 25 & 75 & -- & --- & --- \\
\hline CASE SPARSE OCOSCE & 29 & 180 & -- & --- & -- & - & 75 & 25 & --- \\
\hline CASCIA & 277 & 20 & 48 & 48 & 25 & 25 & 35 & 15 & -- \\
\hline CASCINE DI OPAGNA & 9 & 180 & -- & -- & --- & 10 & 90 & --- & --- \\
\hline CASTEL S.GIOUANNI & 14 & 60 & 30 & 16 & 10 & 45 & 65 & 10 & --- \\
\hline $\begin{array}{l}\text { CASTEI. S. MARIA } \\
\text { CERASOLA }\end{array}$ & $\begin{array}{l}63 \\
24\end{array}$ & $\begin{array}{r}90 \\
---\end{array}$ & $\begin{array}{r}9 \\
100\end{array}$ & $\begin{array}{r}1 \\
---\end{array}$ & $---\overline{26}$ & $-\frac{-15}{25}$ & $\begin{array}{l}45 \\
50\end{array}$ & $\begin{array}{r}56 \\
5\end{array}$ & $\begin{array}{r}5 \\
--\end{array}$ \\
\hline CHIAVANI & $\begin{array}{l}24 \\
51\end{array}$ & 69 & $\begin{array}{r}160 \\
38\end{array}$ & 1 & $-2 b$ & -23 & 45 & 58 & 5 \\
\hline & 48 & 95 & 5 & $-\ldots$ & --- & --- & 45 & 56 & 5 \\
\hline COLFORCEI LA & 29 & 38 & 50 & 2 & 20 & 45 & 30 & 5 & --- \\
\hline COILLE OI AVENDITA & 13 & 15 & 70 & $1 \overline{5}$ & 10 & 20 & 55 & 45 & --- \\
\hline COI.LELII ACONE & 3 & --- & -- & 190 & --- & 180 & --- & --- & --- \\
\hline COI_LE. S.STEF ANO & 2 & 100 & -- & --- & --- & --- & --- & -- & -- \\
\hline COLFOTINO & 14 & 95 & $-\infty$ & 5 & -- & 20 & 58 & 30 & -- \\
\hline NFLI A & 48 & 99 & --- & 4 & -- & $-\infty$ & 25 & 75 & --- \\
\hline FOGLIANO & 54 & 25 & 60 & 15 & -- & 10 & 60 & 30 & --- \\
\hline FUSTAGNA & 14 & 180 & -- & --- & --- & 10 & 70 & 20 & --- \\
\hline GIAPPIENI & 25 & 5 & 15 & 80 & --- & 10 & 20 & 70 & --- \\
\hline LOGNA & 34 & 45 & 80 & 5 & 40 & 35 & 20 & 5 & -- \\
\hline MACERILI 0 & 3 & 100 & --- & -- & -- & 50 & 50 & $-\infty$ & $-\infty$ \\
\hline MALT SGNANO & 57. & 58 & 46 & 10 & --- & 10 & 40 & 56 & --- \\
\hline MANIGI & 17 & 45 & 58 & 5 & --- & 15 & 75 & 10 & --- \\
\hline oco & 58 & 15 & 75 & 10 & 15 & 60 & 20 & 5 & -- \\
\hline ONE & 44 & 15 & 80 & 5 & 35 & 35 & 38 & -- & -- \\
\hline$O P A$ & 7 & 108 & -- & -- & $2 \bar{\theta}$ & $-\cdots$ & 50 & 30 & -- \\
\hline PAOULE & 3 & 100 & --- & --- & -- & 56 & 56 & -- & --- \\
\hline PALMAIOLO & 14 & 75 & 15 & 19 & 10 & 20 & so & 20 & --- \\
\hline POGGIO PRIMUCASO & 17 & $\mathbf{5}$ & 25 & $7 \varepsilon$ & $\mathbf{s}$ & 10 & 15 & 78 & --- \\
\hline PLIRO & 8 & 10 & 30 & 68 & -- & 20 & 60 & 20 & --- \\
\hline ROCCAPORENA & 10 & 18 & 90 & --- & 48 & 10 & 30 & 28 & --- \\
\hline S.ANATUI IA & 12 & 25 & 25 & 56 & $-\infty$ & 38 & 60 & 18 & --- \\
\hline S.GIORGIO & 35 & 47 & $8 \theta$ & 3 & $-\infty$ & 56 & 45 & $\mathbf{5}$ & --- \\
\hline S.TRINITA & 8 & 100 & -- & -- & $-\infty$ & -- & 50 & 56 & --- \\
\hline SCIEDI & 30 & 100 & --- & $-\infty$ & 20 & 56 & 45 & 15 & --- \\
\hline SERUIGI IO & 6 & 100 & -- & -- & -- & -- & 25 & 75 & $-\cdots$ \\
\hline TAZ7O & 8 & 95 & $\mathbf{5}$ & $-\infty$ & -- & 10 & 10 & 80 & -- \\
\hline TROGNANO & 76 & 70 & -- & 36 & 5 & 5 & 15 & 75 & --- \\
\hline UALDONICA & 9 & 100 & --- & --- & $-\infty$ & 20 & 66 & 20 & -- \\
\hline VILLA S.SILUESTRO & 41 & 100 & --- & -- & --- & & 75 & 25 & -- \\
\hline
\end{tabular}

\begin{tabular}{|c|c|c|c|c|c|c|c|c|c|}
\hline 1 & 2 & & 3 & & & & 4 & & \\
\hline & & $A[z]$ & $B(z)$ & $C[\pi]$ & $1[x]$ & $2(x)$ & $3 i z]$ & $4[\%]$ & $5[x]$ \\
\hline $\begin{array}{l}\text { BORGO CERRETO } \\
\text { BUGGIANI } \\
\text { CERRTTO DI SPOLETO } \\
\text { COLLESOGLIO } \\
\text { FFRGINI (ALTO-BASSOJ } \\
\text { NURTOSEF. } \\
\text { PONTE } \\
\text { PONTE DEL PIANO } \\
\text { ROCCHETTA } \\
\text { S.SAUINO } \\
\text { TRIPONZI }\end{array}$ & $\begin{array}{r}78 \\
16 \\
105 \\
11 \\
5 \\
46 \\
5.2 \\
8 \\
39 \\
12 \\
56\end{array}$ & $\begin{array}{r}75 \\
100 \\
60 \\
106 \\
100 \\
90 \\
90 \\
100 \\
80 \\
180 \\
70\end{array}$ & $\begin{array}{r}25 \\
--\frac{25}{40} \\
--- \\
-10 \\
10 \\
--- \\
24 \\
-30 \\
30\end{array}$ & $\begin{array}{l}--- \\
--- \\
--- \\
--- \\
--- \\
-- \\
-- \\
-- \\
-- \\
--\end{array}$ & $\begin{array}{l}30 \\
48 \\
60 \\
58 \\
48 \\
30 \\
30 \\
45 \\
55 \\
40 \\
40\end{array}$ & $\begin{array}{r}16 \\
31 \\
30 \\
40 \\
-10 \\
16 \\
25 \\
35 \\
25 \\
68 \\
20\end{array}$ & $\begin{array}{r}50 \\
30 \\
10 \\
10 \\
60 \\
40 \\
25 \\
10 \\
20 \\
-- \\
30\end{array}$ & $\begin{array}{r}10 \\
--- \\
--- \\
--- \\
20 \\
20 \\
16 \\
--- \\
--10\end{array}$ & $\begin{array}{l}-- \\
--- \\
--- \\
--- \\
--- \\
--- \\
-- \\
-- \\
---\end{array}$ \\
\hline
\end{tabular}




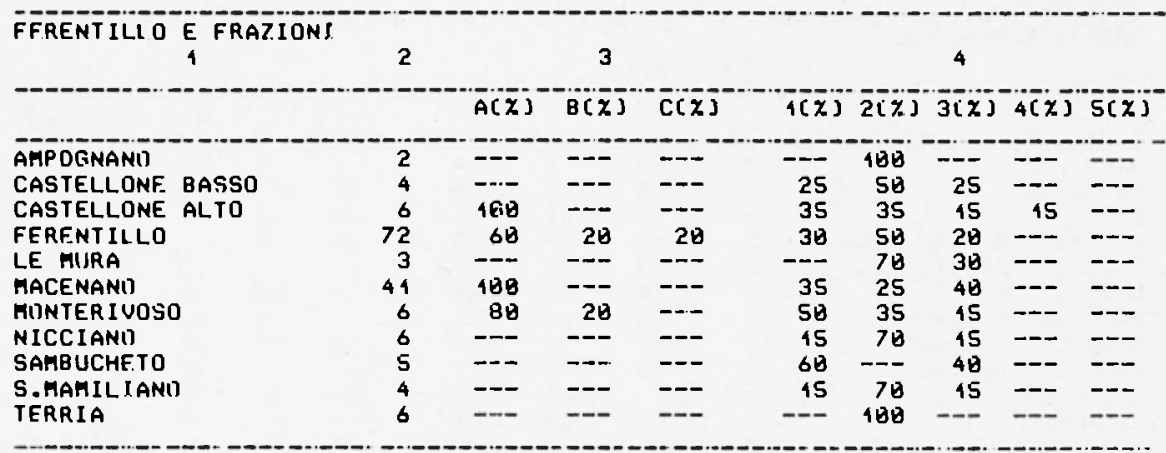

MONTELFUNF, DI SPOLETO E FRAZIOHI 2

3 4

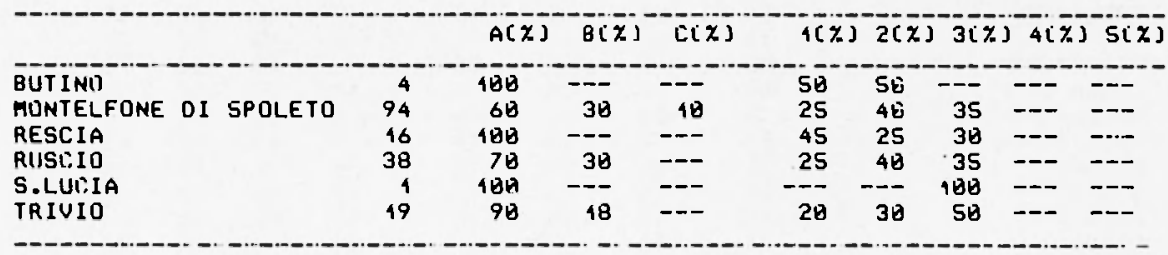

\begin{tabular}{|c|c|c|c|c|c|c|c|c|c|}
\hline 4 & 2 & & 3 & & & & 4 & & \\
\hline & & $A(x)$ & $B(x)$ & $c(x)$ & $f(x)$ & $2(\%)$ & $3 i \geqslant i$ & $4(\%)$ & $\operatorname{six}(x)$ \\
\hline AGR SANI & 104 & 80 & 20 & --- & 45 & 20 & 68 & 5 & \\
\hline ALIENA & 56 & 80 & 26 & -- & 40 & 25 & 60 & 5 & --- \\
\hline ANCARANU & 24 & 100 & $-\infty$ & --- & 45 & 66 & 25 & -- & --- \\
\hline BISELLI & 16 & 90 & 16 & --- & 40 & 5 & 45 & $4 \theta$ & -- \\
\hline CAMP I & 42 & RS & 15 & --- & 45 & 60 & 15 & 18 & -- \\
\hline CASAI I OI SFRRAUAI LE & 24 & 70 & 36 & --- & 45 & 10 & 45 & -- & -- \\
\hline CASTEI LULC IO & 43 & $48 B$ & -- & --- & 5 & 75 & -- & 26 & -- \\
\hline COR I I GNANU & 48 & 4kiv & -- & --- & 5 & --- & 85 & 16 & --- \\
\hline FORSIUO & 38 & 80 & $2 \mathbf{b}$ & --- & --- & 18 & 75 & 15 & -- \\
\hline FRAST.ARO & 49 & 70 & 30 & --- & 48 & 78 & 20 & $\ldots$ & --- \\
\hline LEGOGNE & 48 & 90 & 40 & --- & 5 & --- & 85 & 10 & -- \\
\hline MURCIA (ZONA I] & 84 & 60 & 48 & --- & 30 & 35 & 38 & 5 & --- \\
\hline NURCIA (ZONA 2$]$ & 195 & 50 & 59 & -- & 30 & 39 & 36 & 48 & --- \\
\hline NURCIA (ZONA 3) & 450 & 70 & 28 & 10 & 45 & 25 & 25 & 5 & -- \\
\hline NURCIA (ZONA 4) & 133 & 50 & 48 & 4日 & 45 & 25 & 38 & -- & -- \\
\hline NURCIA (ZOHA S) & 89 & 60 & $4 \overline{0}$ & --- & 40 & 15 & $4 \theta$ & 5 & --- \\
\hline NURCIA (ZONA 6) & 422 & 68 & $4 \theta$ & --- & 48 & 20 & 40 & -- & --- \\
\hline NIITTOR IA & 72 & 78 & 38 & $-\cdots$ & 45 & 45 & 35 & 5 & --- \\
\hline OCR I CCHIO & 24 & RS & 15 & --- & 5 & 48 & 20 & 35 & ---- \\
\hline OSPEDALETTO & 25 & 70 & 38 & --- & 20 & 30 & 45 & 5 & -- \\
\hline PAGANELL I & 19 & $B B$ & 20 & $\cdots$ & 5 & 5 & 60 & 30 & --- \\
\hline PESCIA & 63 & 80 & 28 & $\cdots-$ & 10 & 30 & 50 & 10 & --- \\
\hline PIEOIRIPA & 29 & 80 & 28 & $-\cdots$ & 20 & 40 & 26 & 20 & -- \\
\hline POPOI.I & 48 & 180 & -- & $\cdots-$ & 5 & 35 & 50 & 10 & --- \\
\hline 5. ANDREA & 27 & 85 & 15 & --- & 5 & 26 & 30 & 45 & --- \\
\hline S. MARCO & 44 & 180 & --- & --- & --- & 10 & 78 & 26 & $-\cdots$ \\
\hline S.PEL LF LRINI] & 165 & 1RA & --- & --- & 50 & 30 & 15 & 5 & --- \\
\hline SAUEL.LI & 94 & 65 & 36 & 40 & $\mathbf{5}$ & 15 & 75 & 5 & $-\cdot-$ \\
\hline SFRRAVAI.LE. & 54 & 88 & 20 & $-\infty$ & 10 & 15 & 75 & --- & -- \\
\hline VALCALDARA & 66 & 80 & 20 & -- & 15 & 25 & $4 \theta$ & $2 \theta$ & --- \\
\hline
\end{tabular}




\begin{tabular}{|c|c|c|c|c|c|c|c|c|c|}
\hline PUOSTUDUMU & F NHLIUN & & 3 & & & & 4 & & \\
\hline & & $A[z]$ & $B[z]$ & $C[z]$ & $q(z)$ & $2(z)$ & $3(z)$ & $4[x]$ & $s(x)$ \\
\hline $\begin{array}{l}\text { MUCCIAFORA } \\
\text { POGGIODOMO } \\
\text { ROCCATAMBURO } \\
\text { USIGNJ. }\end{array}$ & $\begin{array}{l}32 \\
38 \\
29 \\
30\end{array}$ & $\begin{array}{l}75 \\
40 \\
70 \\
75\end{array}$ & $\begin{array}{l}25 \\
60 \\
30 \\
25\end{array}$ & $\begin{array}{l}--- \\
--- \\
---\end{array}$ & $\begin{array}{l}45 \\
65 \\
10 \\
75\end{array}$ & $\begin{array}{r}35 \\
35 \\
5 \\
20\end{array}$ & $\begin{array}{r}25 \\
-85 \\
5\end{array}$ & $\begin{array}{l}--- \\
--- \\
---\end{array}$ & $\begin{array}{l}--- \\
--- \\
---\end{array}$ \\
\hline
\end{tabular}

\begin{tabular}{|c|c|c|c|c|c|c|c|c|c|}
\hline \multirow{2}{*}{ PRECI E FRAZIOHI } & \multirow[t]{2}{*}{2} & \multicolumn{3}{|c|}{3} & \multicolumn{5}{|c|}{4} \\
\hline & & $A[z]$ & $B[z]$ & $C[\boldsymbol{x}]$ & $4(x)$ & $2(x)$ & $3[x]$ & $4(z)$ & $5(\bar{x})$ \\
\hline ABETO & 22 & $7 \bar{\theta}$ & 30 & $=$ & 45 & 40 & 35 & 40 & -- \\
\hline BELFORTE-S.LAZZARO & 49 & 80 & 20 & -- & 40 & 20 & 40 & --- & - \\
\hline CASTEL VECCHIO & 44 & 80 & 26 & --- & 45 & 45 & 40 & --- & -- \\
\hline CERUARA & 2 & - & -- & -- & 100 & $\ldots$ & -- & $-\infty$ & - \\
\hline COLLAZZUNI-C.IVITEI LA & 43 & 88 & 20 & --- & 20 & 30 & 25 & 25 & -- \\
\hline COLI ESCILI.E. & 44 & 80 & 20 & --- & 60 & 38 & 10 & --- & - \\
\hline CORMNE & 25 & $8 B$ & 20 & --- & 48 & 48 & 20 & --- & -- \\
\hline FIANO & 20 & 90 & 18 & -- & -- & 25 & 50 & 25 & -- \\
\hline MONTAGLIONF & 47 & 88 & 20 & $-\infty$ & 25 & 20 & 20 & 35 & $\ldots$ \\
\hline MUNTERUFO & 445 & 98 & 10 & $--\infty$ & --- & --- & $4 ?$ & $9 \theta$ & -- \\
\hline PIEDIUAI_LF & 46 & 60 & 30 & 40 & 48 & 20 & 40 & --- & -- \\
\hline PRECI (ALTO E BASSO) & 39 & 68 & 30 & $(40)$ & 70 & 10 & 20 & -- & -- \\
\hline ROCCANUI FI & 57 & 813 & 28 & --- & 35 & 40 & 25 & --- & --- \\
\hline SACCOUESCIO & 23 & 70 & 38 & -- & 80 & 5 & 45 & --- & --- \\
\hline S.VITO & 7 & 89 & 20 & --- & 60 & $4 \bar{\theta}$ & --- & --- & -- \\
\hline TODIANO & 54 & 80 & 20 & --- & 40 & 35 & 25 & 30 & -- \\
\hline VALLE ACQUARO & 2 & 400 & -- & $-\infty$ & --- & 100 & -- & -- & -- \\
\hline VILLA CAMPANARA & 8 & 490 & --- & $-\infty$ & 40 & -- & 40 & 89 & --- \\
\hline UILI.A DEL GUADO & 6 & $4 \in \theta$ & --- & --- & 70 & 15 & 45 & -- & --- \\
\hline
\end{tabular}

\begin{tabular}{|c|c|c|c|c|c|c|c|c|c|}
\hline 4 & 2 & & 3 & & & & 4 & & \\
\hline & & $A(\boldsymbol{Z})$ & $B[z]$ & $C(\bar{z})$ & $\{[X]$ & $2(z)$ & 3[\rangle$\left._{2}\right]$ & $4(x)$ & siz? \\
\hline $\begin{array}{l}\text { CASO } \\
\text { CASTEI. S.FEI.ICE } \\
\text { GAUELI.I } \\
\text { GROTTI } \\
\text { S.ANATOI. IA DI NARCO } \\
\text { S.MARTINO }\end{array}$ & $\begin{array}{r}35 \\
34 \\
15 \\
8 \\
64 \\
14\end{array}$ & $\begin{array}{r}80 \\
90 \\
80 \\
70 \\
70 \\
180\end{array}$ & $\begin{array}{r}20 \\
10 \\
20 \\
30 \\
30 \\
----\end{array}$ & $\begin{array}{l}--- \\
--- \\
--- \\
--- \\
--\end{array}$ & $\begin{array}{r}35 \\
75 \\
108 \\
70 \\
78 \\
70\end{array}$ & $\begin{array}{r}65 \\
25 \\
-- \\
28 \\
28 \\
38\end{array}$ & $\begin{array}{r}--- \\
18 \\
---\end{array}$ & $\begin{array}{l}--- \\
--- \\
--- \\
--- \\
---\end{array}$ & $\begin{array}{l}--- \\
--- \\
--- \\
--- \\
--\end{array}$ \\
\hline
\end{tabular}




\begin{tabular}{|c|c|c|c|c|c|c|c|c|c|}
\hline SCHFIIGINO E FRATIONI & 2 & & 3 & & \multicolumn{4}{|c|}{4} & \\
\hline & & $A[x]$ & $8(x)$ & $C(\geqslant)$ & $i[x]$ & $\overrightarrow{2}(\bar{x})$ & $3(x)$ & $4(\bar{x})$ & $5(x)$ \\
\hline CFSFLLI & 18 & 80 & 20 & --- & 5 & $4 B$ & 5 & 5 & -- \\
\hline CIUITEI.I A & 18 & 980 & -- & --- & 25 & 35 & 45 & 25 & -- \\
\hline COLI_FFARR I & 1 & -- & --- & --- & --- & -- & 100 & -- & -- \\
\hline MONTE S.UITO & 20 & 100 & $-\infty$ & -- & 85 & 48 & 5 & -- & -- \\
\hline PUNTUGLIA & 4 & -- & --- & -- & -- & -- & 25 & 75 & -- \\
\hline S. VAL ENT INO & 6 & 90 & $4 \theta$ & -- & --- & 75 & 25 & --- & -- \\
\hline SCHEGG INO & 45 & 68 & 48 & -- & 35 & 60 & 5 & --- & --- \\
\hline
\end{tabular}

\begin{tabular}{|c|c|c|c|c|c|c|c|c|c|}
\hline 1 & 2 & & 3 & & & & 4 & & \\
\hline & & $A(x)$ & $B(x)$ & $C(\%)$ & $4(\geqslant)$ & $2(z)$ & $3[z]$ & $4(x)$ & $5(\%)$ \\
\hline APAGNI & 2 & -- & -- & $=-$ & -- & 56 & 50 & $=-$ & -- \\
\hline CAMMIIRO & 10 & $\tan$ & -- & $\ldots$ & $2 \theta$ & 10 & 70 & -- & -- \\
\hline LFTERNF & 5 & $-\ldots$ & --- & --- & 20 & 20 & 60 & --- & --- \\
\hline MOLINI & 10 & --- & --- & --- & 100 & $--\infty$ & --- & -- & --- \\
\hline MUNTALBO & 48 & 180 & -- & --- & 28 & 58 & 30 & -- & -- \\
\hline MUNTESANTO & 6 & 180 & -- & --- & -- & $2 \theta$ & 86 & -- & -- \\
\hline NUCAL. I & $?$ & -- & -- & --- & 48 & 80 & 18 & -- & -- \\
\hline URSANU & 48 & 181 & -- & --- & 59 & 38 & $2 B$ & -- & -- \\
\hline POSTIGNANO E COLLE & 9 & 100 & -- & -- & 45 & 16 & 45 & -- & $-\cdots$ \\
\hline PUPAGG I & 10 & 90 & 18 & --- & 50 & 39 & 28 & --- & --- \\
\hline SEI L ANI & 67 & 60 & 30 & 18 & 30 & $2 \theta$ & 45 & 5 & --- \\
\hline STERPARE & 7 & 100 & -- & -- & 10 & -- & 90 & -- & -- \\
\hline TRIOBIO & 6 & -- & --- & --- & 38 & 20 & 50 & --- & --- \\
\hline UILI_AFALINA E CASAI_E & 46 & 70 & 20 & 19 & 25 & 56 & 25 & -- & -- \\
\hline
\end{tabular}

\begin{tabular}{|c|c|c|c|c|c|c|c|c|c|}
\hline \multirow[b]{2}{*}{ 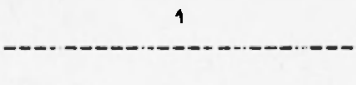 } & 2 & & 3 & & & & 4 & & \\
\hline & & $A(\bar{x})$ & $\theta(x)$ & $C(\pi)$ & $4(\%)$ & $2(x)$ & $3(x)$ & $A(x)$ & $5(\%)$ \\
\hline GEPPA & 5 & 108 & --- & $=-$ & 48 & 28 & 40 & $--\infty$ & --- \\
\hline MEGG I ANI & 63 & $7 \theta$ & $3 \mathrm{~B}$ & -- & $2 A$ & 35 & 45 & --- & --- \\
\hline MUNTEF IORFLL O & 34 & 89 & 28 & --- & SB & $2 \theta$ & $3 \bar{E}$ & --- & --- \\
\hline PATERNO & 14 & 90 & 19 & -- & $A B$ & $2 B$ & 48 & --- & --- \\
\hline PIEOILACISTA & 13 & 180 & $-\infty$ & --- & 25 & 39 & 45 & --- & -- \\
\hline$P$ IENIPATERNI,$L^{\prime}$ EREMITA & 84 & 89 & 28 & --- & 50 & 25 & 25 & $-\infty$ & --- \\
\hline PONTEBORBOUNF:A & 24 & --- & --- & --- & 5 & 95 & -- & -- & $-\infty$ \\
\hline PRATALE & $i$ & --- & --- & -- & $19 \bar{B}$ & -- & --- & --- & --- \\
\hline VALI.O DI NFRA & 146 & 80 & 20 & --- & $A B$ & 25 & 35 & --- & --- \\
\hline
\end{tabular}




\section{BIBLIOGRAFIA}

Baratta M., 1901 - I terremoti d'llalia. F.lli Bocca, Torino.

BLAKE A., 1941 - On the estimation of focal depth from macroseismic data. "Boll. Seism. Soc. Am.", 31, 3, pp. 225-231.

Boccaletti M., Coli M., Napoleone G., 1977 - Nuovi allineamenti da immagini LAND-SAT e rapporti con l'attività sismica negli Appennini. «Boll. Soc. Geol. It. », 96, p. 679-694.

Cagnetti V., Pasounle V., Polinari S., 1978 - Fault plane solutions and stress regime in Italy and adjacent regions. "Tectonophysics", 46, pp. 239-250.

Calamita F., Cantalamiessa G., Centamore E., Deinna G., Dramis F., Micarelli A., Pierruccini U., Potetti M., Romano A., 1978 - Dati preliminari sulla neotettonica dei Fogli 182 (Norcia), 124 (Macerata; III-IV Quadrante), 115 (Citıà di Castello; I-II Quadrante). Nuovi contributi alla realizzazione della carla neotettonica d'Italia. Pubbl. 251 P.F.G. pp. 179-215.

Calor P., 1942 - Attività sismica in Italia nel decentio 1930-1939. Reale Acc. d'It., IX, F. Le Monnier - Firenze.

CIIANDra U., 1979 - Attentation of intensities in the United States. "Bull. Seism. Soc. Am. ", 69, 6, pp. 2003-2024.

Console R., Peronaci F., Sonaglia A., 1971 - Fenomeni sismici a Mignano Montelungo. "Ann. di Geof.", XXIV, 1, pp. 55-88.

Console R., Sonaglia A., 1972 - Studio del terremoto di Tuscania. "Ann. di Geof., «XXV, 3, pp. 360-380.

De Panfilis M., 1958 - Attività sismica in Italia dal 1953 al 1957. "Ann. di Geof. ", XII, 1, pp. 3-130.

Gasparini C., 1976 - Parametri ipocentrali dei dati macrosismici del ter. remoto del Friuli, maggio 1976. "Ann. di Geof.", XXIX, 3, pp. 159-164.

Marcelli L., Spadea M.C., 1981 - Ricerca del coefficiente $\gamma=f(h)$ (della formula di Blake) valevole per il territorio italiano. Metodo rapido per calcolare "l macrosismica" con le prime tre isosiste di tin terremoto. "Ann. di Geof."XXXIV, in press. 
Ogniben L., Parotto M., Praturion A., 1975 - Structural model of Italy. Quaderni Ricerca Scientifica, C.N.R., 90.

Scarslilda M., 1941 - Carta Geologica d'Italia. Foglio 132 (Norcia) - Servizio Geologico d'Italia.

Vi:Red M., StrieM H.L., 1977 - A macroseismic study and the implication of structural damage of two recent earthquakes in the Jordan rift. "Bull. Seism. Soc. Am.", 67, 6, pp. 1607-1613. 